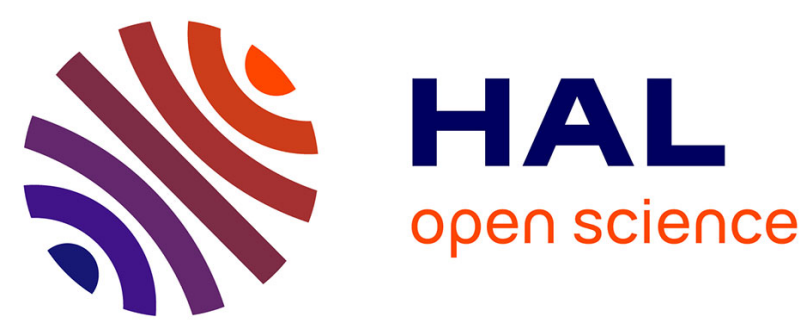

\title{
Insoluble organic matter in chondrites: Archetypal melanin-like PAH-based multifunctionality at the origin of life?
}

Marco d'Ischia, Paola Manini, Zita Martins, Laurent Remusat, Conel O'D. Alexander, Cristina Puzzarini, Vincenzo Barone, Raffaele Saladino

\section{To cite this version:}

Marco d'Ischia, Paola Manini, Zita Martins, Laurent Remusat, Conel O'D. Alexander, et al.. Insoluble organic matter in chondrites: Archetypal melanin-like PAH-based multifunctionality at the origin of life?. Physics of Life Reviews, 2021, 37, pp.65-93. 10.1016/j.plrev.2021.03.002 . hal-03206027

\section{HAL Id: hal-03206027 https://hal.science/hal-03206027}

Submitted on 22 Apr 2021

HAL is a multi-disciplinary open access archive for the deposit and dissemination of scientific research documents, whether they are published or not. The documents may come from teaching and research institutions in France or abroad, or from public or private research centers.
L'archive ouverte pluridisciplinaire HAL, est destinée au dépôt et à la diffusion de documents scientifiques de niveau recherche, publiés ou non, émanant des établissements d'enseignement et de recherche français ou étrangers, des laboratoires publics ou privés. 


\section{Physics of Life Reviews}

Insoluble Organic Matter in Chondrites: Archetypal Melanin-Like PAH-Based Multifunctionality at the Origin of Life?

--Manuscript Draft--

\begin{tabular}{|c|c|}
\hline Manuscript Number: & \\
\hline Keywords: & $\begin{array}{l}\text { insoluble organic matter; melanins; polycyclic aromatic hydrocarbons (PAHs); } \\
\text { Primordial Multifunctional organic Entity (PRiME) }\end{array}$ \\
\hline Corresponding Author: & $\begin{array}{l}\text { Marco d'Ischia } \\
\text { University of Naples Federico II } \\
\text { Naples, ITALY }\end{array}$ \\
\hline First Author: & Marco d'Ischia \\
\hline \multirow[t]{5}{*}{ Order of Authors: } & Marco d'Ischia \\
\hline & Paola Manini \\
\hline & Cristina Puzzarini \\
\hline & Vincenzo Barone \\
\hline & Raffaele Saladino \\
\hline
\end{tabular}




\section{Highlights}

- The IOM in chondrites is remarkably similar to the allomelanins in microorganisms.

- Allomelanins can promote phenomena and processes of astrobiological relevance.

- Being similar to allomelanins, IOM may have played a role in the origin of life. 


\title{
Insoluble Organic Matter in Chondrites: Archetypal Melanin-Like PAH-Based
}

\section{Multifunctionality at the Origin of Life?}

Marco d'Ischia ${ }^{1}$, Paola Manini ${ }^{1}$, Zita Martins ${ }^{2}$, Laurent Remusat ${ }^{3}$, Conel M. O’D. Alexander ${ }^{4}$, Cristina Puzzarini $^{5}$, Vincenzo Barone ${ }^{6}$ and Raffaele Saladino ${ }^{7}$

${ }^{1}$ Department of Chemical Sciences, University of Naples Federico II, Via Cintia 4, 80126 Naples, Italy ${ }^{2}$ Centro de Química Estrutural and Departamento de Engenharia Química, Instituto Superior Técnico, Universidade de Lisboa, Av. Rovisco Pais 1, 1049-001 Lisboa, Portugal

${ }^{3}$ Institut de minéralogie, de physique des matériaux et de cosmochimie, UMR CNRS 7590, Sorbonne Université, Muséum National d'Histoire Naturelle, 61 rue Buffon, 75005 Paris, France

${ }^{4}$ Earth and Planets Laboratory, Carnegie Institution for Science, 5241 Broad Branch Road, NW Washington, DC 20015-1305, USA

${ }^{5}$ Department of Chemistry “Giacomo Ciamician”, University of Bologna, Via F. Selmi 2, Bologna, I40126, Italy

${ }^{6}$ Scuola Normale Superiore, Piazza dei Cavalieri 7, Pisa, I-56126, Italy

${ }^{7}$ Biological and Ecological Sciences Department (DEB), University of Tuscia, Via S. Camillo de Lellis 01100 Viterbo, Italy

\begin{abstract}
An interdisciplinary review of the chemical literature that points to a unifying scenario for the origin of life, referred to as the Primordial Multifunctional organic Entity (PriME) scenario, is provided herein. In the PriME scenario it is suggested that the Insoluble Organic Matter (IOM) in carbonaceous chondrites, as well as interplanetary dust particles from meteorites and comets may have played an important role in the three most critical processes involved in the origin of life, namely 1) metabolism, via $a$ ) the provision and accumulation of molecules that are the building blocks of life, $b$ ) catalysis
\end{abstract}


(e.g., by templation), and $c$ ) protection of developing life molecules against radiation by excited state deactivation; 2) compartmentalization, via adsorption of compounds on the exposed organic surfaces in fractured meteorites, and 3) replication, via deaggregation, desorption and related physical phenomena. This scenario is based on the hitherto overlooked structural and physicochemical similarities between the IOM and the dark, insoluble, multifunctional melanin polymers found in bacteria and fungi and associated with the ability of these microorganisms to survive extreme conditions, including ionizing radiation. The underlying conceptual link between these two materials is strengthened by the fact that primary precursors of bacterial and fungal melanins (collectively referred to herein as allomelanins) are hydroxylated aromatic compounds like homogentisic acid and 1,8dihydroxynaphthalene, and that similar hydroxylated aromatic compounds, including hydroxynaphthalenes, figure prominently among possible components of the organic materials on dust grains and ices in the interstellar matter, and may be involved in the formation of IOM in meteorites. Inspired by this rationale, a vis-à-vis review of the properties of IOM from various chondrites and nonnitrogenous allomelanin pigments from bacteria and fungi is provided herein. The unrecognized similarities between these materials may pave the way for a novel scenario at the origin of life, in which IOM-related complex organic polymers delivered to the early Earth are proposed to serve as PriME and were preserved and transformed in those primitive forms of life that shared the ability to synthesize melanin polymers playing an important role in the critical processes underlying the establishment of terrestrial eukaryotes.

Keywords: insoluble organic matter; melanins; polycyclic aromatic hydrocarbons (PAHs); Primordial Multifunctional organic Entity (PRiME) 
Table of contents

\section{Introduction}

2. Melanin-type materials and precursors in astrochemical environments

3. Insoluble Organic Matter from chondrites and comets

3.1. Definition and occurrence

3.2. Structural and physical properties

3.3. Origin

3.4. Formation pathways of putative IOM precursors and aromatic compounds

4. Melanins as evolutionarily preserved complex multifunctional organic polymers

4.1. Classification, occurrence and physicochemical properties

4.2. Biological roles and functions

5. The role of IOM in prebiotic processes: from melanin connection to the PriME scenario

5.1. Structural and physicochemical similarities between IOM and allomelanin

5.2. Chemical requirements at the origin of life

5.3. The PriME scenario and the Universal Multifunctional Adaptive Mediator paradigm

5.4. The PriME scenario and Virtual Reality

6. Conclusions and perspectives 


\section{Introduction}

Meteorites, with the exception of a few samples from Mars and the Moon, are fragments of asteroids from the asteroid belt located between Mars and Jupiter. The chondritic meteorites are the most primitive type of meteorite having avoided the melting and differentiation that affected other meteorite types. The heating of planetesimals in the early Solar System was mostly driven by the decay of the short-lived radionuclides ${ }^{26} \mathrm{Al}\left(\mathrm{t}_{1 / 2} \approx 0.7 \mathrm{Ma}\right)$ [1]. The chondrite parent bodies formed between $\sim 2 \mathrm{Ma}$ and $\sim 4 \mathrm{Ma}$ after Solar System formation when there was still enough ${ }^{26} \mathrm{Al}$ to drive rock-forming geological processes (lithification), such as thermal metamorphism and aqueous alteration, but not enough to melt them. Based on their bulk compositions and physical properties, the chondrites have been subdivided into a number of classes and groups: enstatite (EH and EL), Rumuruti, ordinary (H, L and LL), and carbonaceous (CI, CM, CO, CV, CK, CR, CH and CB) [2-5]. Chondrites are also assigned petrologic types (e.g., CM1 and LL6) depending on the extent of aqueous alteration (1-3, with 1 being the most aqueously altered, and 3 the least) or thermal metamorphism (3-6, with 6 being the one that experienced the most thermal metamorphism) they experienced. Carbonaceous chondrites are a pristine class of materials that formed from the protoplanetary disks and together with interplanetary dust particles (IDPs) from meteorites and comets and Ultra-Carbonaceous Antarctic Micrometeorites (UCAMMs) provided the major source of organic carbon in the early Earth.

Chondrites contain inclusions (chondrules and refractory inclusions) set in a fine-grained matrix. It is in the matrix that one finds the most primitive materials, such as presolar circumstellar grains and organic matter. The circumstellar grains are identified based on their large isotope anomalies that can only be explained by nucleosynthetic processes. Ices also seem to have been important components of the matrices of all chondrites, except the highly reduced enstatite chondrites. At the time of the formation of their parent bodies, the matrices of all chondrites may have contained $\sim 3-4$ wt. $\%$ of organic carbon. This organic material is generally divided in a complex suite of solvent Soluble Organic Matter (SOM) and a more abundant macromolecular component, the Insoluble Organic Matter (IOM). IOM is a 
descriptive term that has been widely adopted to indicate the larger portion (75-95\%) of the organic carbon present in chondrites $[6,7]$. The remaining $\%$ of the organic matter in carbonaceous chondrites is composed of a mixture of solvent-soluble organic compounds (i.e., SOM) [8-10]. The organic material in most chondrites has been heavily modified or destroyed by thermal metamorphism and shock heating. However, members of the CI, CM and CR groups experienced variable degrees of low temperature $\left(<100-150^{\circ} \mathrm{C}\right)$ aqueous alteration. The extent to which aqueous alteration has modified the SOM and IOM remains controversial. Nevertheless, the IOM and many soluble organic compounds in these meteorites have large excesses in deuterium, ${ }^{13} \mathrm{C}$ and ${ }^{15} \mathrm{~N}$ [11-14]. D and ${ }^{15} \mathrm{~N}$ enrichments suggest that they or their precursors formed in low temperature $(10-40 \mathrm{~K})$ and radiation-rich environments in the interstellar medium (ISM) or the outer Solar System [15-23]. Also, CI- and/or CM-like materials are the leading candidates for the major sources of volatiles (e.g., hydrogen, carbon and nitrogen) in the early Earth. If some of this material was delivered by chondritic IDPs, UCAMMs, micrometeorites and meteorites, then they would have also been a significant source of complex, prebiotic organic matter.

Due to its insolubility, IOM has been difficult to characterize in detail at the molecular level and is known mostly through spectroscopic, physical and chemical/pyrolytic analyses. IOM-like materials are also present in various geochemical contexts, such as sedimentary rocks, and are referred to as kerogens.

Despite increasing interest in chondrites as rich sources of soluble and volatile organic compounds of possible relevance to the origin of life, relatively little attention has been devoted to its much more abundant insoluble counterpart. Apart from some systematic chemical and structural characterization studies [6], the general attitude in the literature on the origin of life has been to disregard IOM as an essentially inert material and favor the richer and more readily appreciable chemistry of soluble organic compounds.

The aim of this paper is to draw the attention of scientists involved in research on astrobiology and the origin of life to the so far overlooked, yet most relevant observation that most if not all of the key 
structural features and properties of the IOM found in carbonaceous chondrites are shared by melanins, the ubiquitous dark insoluble phenolic polymers found in almost all types of living organisms, including lower level organisms like bacteria and fungi. Such an analogy emerges clearly from a background of scattered observations, a virtually unprecedented integration of astrochemical, astrophysical, organic and physical chemical viewpoints, and a critical perspective of the evolutionary significance of melanin and related complex organic biopolymers. From bacteria to fungi, plants, cephalopods, birds, mammals up to humans, melanins appear to serve a broad variety of roles and functions which can be re-conceptualized and revisited as fulfilling most of the key chemical requirements at the origin of life. Analogies and matching properties between IOM and melanins in bacteria and fungi, both natural and synthetic, are used herein to suggest that IOM may have been an active player in the origin and processing of life molecules and thus may provide the basis to complement and integrate current prebiotic scenarios in the early Earth into a unifying perspective of possible evolutionary relevance.

\section{Melanin-type materials and precursors in astrochemical environments}

The plausibility that an archetypal melanin-like material could have played a significative role in the emergence of molecular complexity is also based on the availability of suitable chemical precursors in astrochemical environments. The chemical precursors of melanins in extant taxa are represented by two class of organic compounds: $i$ ) the aromatic amino acids phenylalanine (Phe) and tyrosine (Tyr) along with the sulfur containing derivative cysteine (Cys) (Fig. 1, panel A), that are involved in the synthesis of nitrogen containing mammalian melanins, eumelanins and pheomelanins, and ii) the aromatic naphthols 2,3,6,8-tetrahydroxynaphthalene, 2,4,8-trihydroxynaphtalene and 1,8-dihydroxynaphtalene (Fig. 1, panel B), involved in the synthesis of fungal allomelanins. Tyrosine is also involved in the synthesis of the bacterial allomelanins, pyomelanins, via the intermediate formation of homogentisic acid. 
A)<smiles>NC(Cc1ccccc1)C(=O)O</smiles>

Phe<smiles>NC(Cc1ccc(O)cc1)C(=O)O</smiles>

Tyr
B)<smiles>Oc1cc(O)c2c(O)cc(O)cc2c1</smiles><smiles>Oc1cc(O)c2c(O)cccc2c1</smiles><smiles>NC(CS)C(=O)O</smiles>

Cys

Fig. 1. Chemical precursors of melanins. Panel A: phenylalanine (Phe), tyrosine (Tyr) and cysteine (Cys) amino acids as chemical precursors of eumelanins and pheomelanins. Panel B: 2,3,6,8-tetrahydroxy naphthalene, 2,4,8-trihydroxynaphtalene and 1,8-dihydroxynaphtalene as chemical precursors of fungal allomelanins.

Besides biological environments, melanin-precursor-forming reactions may also operate in a wide range of space environments that are subject to different kinds of conditions, including irradiation by stellar winds, cosmic rays, as well as thermal heating by radioactive decay [24] and aqueous alteration. Then the question is: are the chemical precursors of melanin abundant enough in space to be compatible with the presence of melanin-type materials in astrochemical environments?

Chemical precursors of melanins have been detected in the SOM of carbonaceous chondrites. The SOM of carbonaceous meteorites has a high molecular diversity (in the order of tens of thousands), with a bulk composition of $\sim \mathrm{C}_{100} \mathrm{H}_{155} \mathrm{O}_{20} \mathrm{~N}_{3} \mathrm{~S}_{3}$, [25]. It includes amino acids, diamino acids, dipeptides, hydrocarbons, alcohols, carboxylic acids, diketopiperazines, sulfonic and phosphonic acids, purines, pyrimidines, sugars and sugar-related compounds, amines, amides, aldehydes, and ketones [8]. The extraterrestrial soluble organic molecules present in carbonaceous chondrites are enriched in ${ }^{13} \mathrm{C}, \mathrm{D}$ and ${ }^{15} \mathrm{~N}$ [14, 26-31], which indicates an interstellar heritage of these molecules or, most likely of their precursors [15-21]. 
Amino acids have been extensively analyzed in carbonaceous chondrites since the 1970's, with more than 80 different amino acids identified (carbon number from $\mathrm{C}_{2}$ through $\mathrm{C}_{9}$ ), and total amino acid abundance of around 60ppm. These have a complete structural diversity, and a decrease in concentration with increasing carbon number [32-36]. While several carbonaceous chondrites were analyzed for amino acids, including CMs, CIs, CRs, CVs, COs, and CKs [37-67], the aromatic amino acids Phe and Tyr are only present in CR chondrites, which are reported in Table $1[68,69]$. To note however, that this last study does not include any procedural blanks or measurement errors, which are crucial to address the accuracy of the measurements [70]. To date, no compound specific isotopic compositions have been performed for Phe or Tyr, and therefore it is not possible to determine without a question whether these two amino acids are indigenous to the carbonaceous chondrites or terrestrial contamination [8].

Table 1. Abundance of phenylalanine and tyrosine in the hydrolyzed, water-extracts of different CR chondrites (nmole/g of meteorite) [104, 105].

\begin{tabular}{lcc}
\hline Meteorite $^{\mathrm{a}}$ & D,L-Phenylalanine & D,L-Tyrosine \\
\hline MET 00426 & 17.8 & 8.0 \\
GRO 95577 & $\leq 1$ & $\leq 1$ \\
MIL 07525 & $\mathrm{nf}^{\mathrm{b}}$ & 12.8 \\
PCA 91082 & $\mathrm{nf}^{\mathrm{b}}$ & $\leq 1$ \\
QUE 99177 & $\mathrm{nf}^{\mathrm{b}}$ & $\leq 1$ \\
EET 92042 & 8.5 & 3.0 \\
GRA 95229 & 24 & 2 \\
LAP 02342 & 127 & $\leq 1$
\end{tabular}

\footnotetext{
${ }^{\mathrm{a}}$ The Renazzo-type (CR) carbonaceous chondrites: Meteorite Hills (MET) 00426, Grosvenor Mountains (GRO) 95577, Miller Range (MIL) 07525, Pecora Escarpment (PCA) 91082, Queen Alexandra Range (QUE) 99177, Elephant Moraine (EET) 92042, Graves Nunataks (GRA) 95229, and LaPaz Icefield

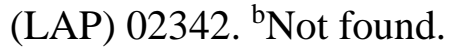


Sulphur containing amino acids have not been observed in carbonaceous chondrites; however, products of sulfhydryl oxidation, such as sulfonic acids and alkyl sulfonates, have been detected in the Murchison meteorite [71], indicating the occurrence of an extensive chemical processing [72].

Seven of the eight possible alkyl sulfonic acids were identified in this CM2 chondrite, with carbon atoms up to $\mathrm{C} 4$, abundances decreasing with increasing carbon number (highest concentration from around 380 nmol/gram) [71]. ${ }^{13} \mathrm{C}$ and D chemical shift values were obtained for alkyl sulfonic acids present in the Murchison meteorites: ${ }^{13} \mathrm{C}$ chemical shift values of $+29.8 \%$, $+9.1 \%,-0.4 \%$, and $-0.9 \%$ for the methyl, ethyl, isopropyl, and n-propyl sulfonic acids, respectively, and D chemical shift values of $+483 \%$, $+787 \%$, $+536 \%$, and $+852 \%$ for the methyl, ethyl, isopropyl, and n-propyl sulfonic acids, respectively [14].

Since the pivotal Miller experiment [73], different prebiotic processes under both space- and planetarylike conditions have been described for the abiotic synthesis of amino acids [74], including Phe, Tyr and Cys derivatives [75]. The abiotic synthesis of Phe and Tyr occurs in a large variety of space and geochemical scenarios, including meteorite-catalyzed thermal processes [76], spark discharge in gas mixtures [77], associated with self-crystallization and racemic resolution [78], high pressure hydrothermal transformations [79], and the geological process of serpentinization [80], also focusing on the catalytic role of biomorph structures [81]. The possible prebiotic synthesis mechanisms for Cys include irradiation [82] and hydrothermal processes [83]. The latter mechanism is generally thought to involve so-called Strecker-cyanohydrin synthesis, a sequential chemical condensation of hydrogen cyanide (HCN) and aldehydes [84]. Recently, the major role of $\mathrm{HCN}$ in the formation of amino acids under spark discharge conditions has been revisited, and formamide $\left(\mathrm{NH}_{2} \mathrm{CHO}\right)$, a widely distributed one-carbon compound in the universe [85], has become the favored intermediate in the process [86]. Examples of the catalytic effects of meteoritic minerals in the prebiotic synthesis of amino acids (and of other biologically relevant molecules) from formamide have been reported for a large variety of physical and chemical conditions. The synthesis could have been fueled by a range of energy sources (thermal, 
solar wind, cosmic rays, radiolysis) [76] when compatible with the stability of the end products [87]. Reductive amination of $\alpha$-keto acids and carbonylation of amines under proton irradiation [88] and Fisher-Tropsch-type (FTT) syntheses [89] have also been proposed as alternative synthetic pathways, both inside and outside (the icy surfaces) [89] the meteorite parent bodies. Although the detailed mechanism(s) for the formation of aromatic amino acids needs to be further explored, a Friedel-Craft type functionalization of a pre-formed benzene ring with acetylene followed by electrophilic addition reactions and hydrolysis has been suggested as possible reaction pathway for the synthesis of Phe and Tyr (Fig. 2) [90].

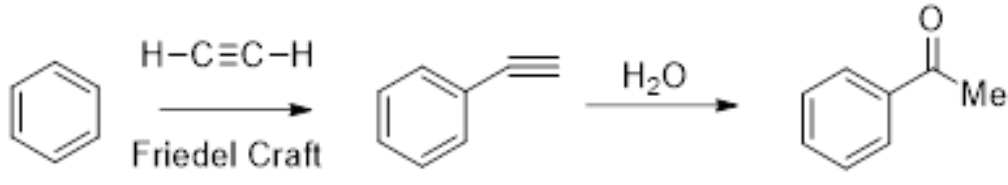
main product<smiles>O=CCc1ccccc1</smiles>
phenylacetaldehyde (by-product)

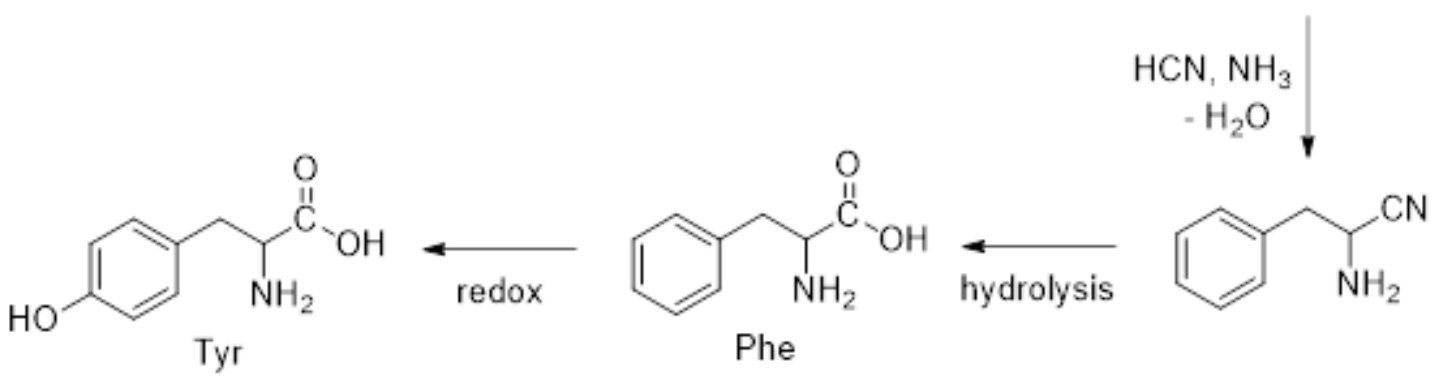

Fig. 2. Abiotic synthesis of phenylalanine (Phe) and tyrosine (Tyr) as suggested by Friedman and Miller [92].

As mentioned previously, allomelanins are mainly produced by oxidative polymerization of DHN, a dihydroxylated derivative of the simplest PAH naphthalene $\left(\mathrm{C}_{10} \mathrm{H}_{8}\right)$ [91]. A large number of PAHs have been detected in carbonaceous chondrites, including naphthalene and alkyl-naphthalene derivatives [55,92-96]. The astrochemical interest in PAHs derives from their detection in various astronomical environments, such as cosmic dust, icy satellites, carbonaceous chondrites, comets. Furthermore, their possible role in prebiotic processes led to introduction of the so-called "PAH world hypothesis" [97]. 
While aromatic naphthols have not been detected in carbonaceous chondrites, naphthalene has been detected in Y-791198 [93], Y-74662 [94] and Murchison [95,96]. Structural isomers of methyl- and dimethylnaphthalene have been detected in Y-791198 [93], and Murchison [95]. The abundances of alkylated PAHs in CM2 chondrites seem to be related to the extension of aqueous alteration on its parent body [55,98]. For example, the Paris meteorite, one of the least aqueously altered carbonaceous chondrite analyzed to date does not contain any alkylated PAHs [55], while other more aqueously altered CM2s (like Murchison and Y-791198) contain alkylated naphthalene.

The Rosetta Orbiter Spectrometer for Ion and Neutral Analysis (ROSINA) analyzed comet 67P/Churyumov-Gerasimenko and detected toluene, but no naphthalene or alkylated naphthalene [99]. The smallest amino acid glycine was also detected in this comet [100], as well as in Wild-2 comet [101,102], with no larger amino acids indisputably being detected. The SOM content of Antarctic micrometeorites (AMMs) has also been analyzed, in particular for PAHs [103,104], and amino acids [105-107]. The AMMs were strongly depleted in naphthalene relative to Murchison [106], and had very low abundances of amino acids [105-107].

\section{Insoluble Organic Matter from chondrites and comets}

\subsection{Definition and occurrence}

The IOM is the major carbonaceous component in chondrites that has been isolated and characterized $[7,10]$. Two basic techniques have been used to demineralize meteorites and concentrate the IOM, one utilizing $\mathrm{HF}-\mathrm{HCl}$ and the other using $\mathrm{CsF}$-dioxane. Both techniques produce similar IOM yields and compositions. However, the IOM recovered in demineralized residues typically only accounts for $\sim 60 \%$ of the bulk carbon [108]. Only a small fraction of the unrecovered carbon can be accounted for by carbonate or solvent extractable organic material. The remainder is probably either in very finegrained IOM that is hard to recover from the demineralizing solutions, or is in acid hydrolysable functional groups that are attached to the IOM. However, at present we cannot exclude that some of 
the missing carbon is present in soluble compounds that are strongly associated with minerals and as a result they cannot be efficiently extracted with solvents prior to demineralization.

In situ observations of carbonaceous grains in matrix, which are presumably dominated by IOM, are free of mineral inclusions and exhibit no obvious spatial relationships with any minerals [109]. The grains have a diversity of morphologies, sizes (mostly $<1 \mu \mathrm{m})$ and isotopic compositions $[109,110]$. These include isolated irregular grains and roughly spherical and often hollow nanoglobules, as well as veins. The veins probably reflect a redistribution of material when ices in the matrix melted and the fluids began to move [111].

This diversity of grain morphologies and isotopic compositions is preserved in demineralized IOM isolates, and it is these residues that have been studied in the most detail. IOM in the CR chondrites appears to be particularly primitive and its bulk elemental composition, $\mathrm{C}_{100} \mathrm{H}_{75-79} \mathrm{O}_{11-17} \mathrm{~N}_{3-4} \mathrm{~S}_{1-3}$ [10], closely resembles the bulk elemental compositions of comet Halley CHON particles, $\sim \mathrm{C}_{100} \mathrm{H}_{80} \mathrm{O}_{20} \mathrm{~N}_{4} \mathrm{~S}_{2}$ [112], and the refractory organics in comet 67P/Churyumov-Gerasimenko dust particles, $\sim \mathrm{C}_{100} \mathrm{H}_{100} \mathrm{~N}_{3}$, measured by the COSIMA instrument on the Rosetta spacecraft $[113,114]$. The refractory organics makes up 30-40 wt.\% of these two comets [115-117], roughly an order of magnitude higher than in chondrite matrices. The CR2s appear to have experienced the most benign alteration conditions for IOM of any chondrite group. The IOM from all other chondrite groups tend to have lower H/C ratios that roughly reflect the intensities of the parent body processing that their host meteorites experienced [10]. However, the extent to which the variations in IOM elemental and isotopic compositions between the most primitive members of each chondrite group were inherited or are the products of parent body processes remains debated [118-120]. The bulk IOM in CR chondrites is also, with rare exceptions, the most isotopically anomalous, both in bulk [10] and at micron to submicron scales $[118,121,122]$.

When analyzed at similar spatial scales, the range of hydrogen and nitrogen isotopic compositions seen in the most primitive IOM is very similar to that seen in the most primitive interplanetary dust particles collected in the stratosphere that may be of cometary origin $[123,124]$. 


\subsection{Structural and physical properties}

Chemical degradation [125-128] and pyrolysis [129-132] studies point to the IOM being composed of small (1-4 ring) polyaromatic units (PAUs) that are highly substituted and cross-linked by short, highly branched aliphatic and ester linkers (Fig. 3). However, yields from both chemical degradation and pyrolysis studies are low, so it is not clear how representative these chemically and thermally accessible components are of the bulk material. In addition, the yields of individual compounds are rarely determined quantitatively. IR spectroscopy suggests that at least the aliphatic material in bulk IOM is indeed composed of short and highly branched carbon units. Based on $\mathrm{RuO}_{4}$ oxidation experiments, it was concluded that the aliphatic material forms bridges between aromatic units [130]. The high degree of cross-linking in IOM likely explains its refractory nature.

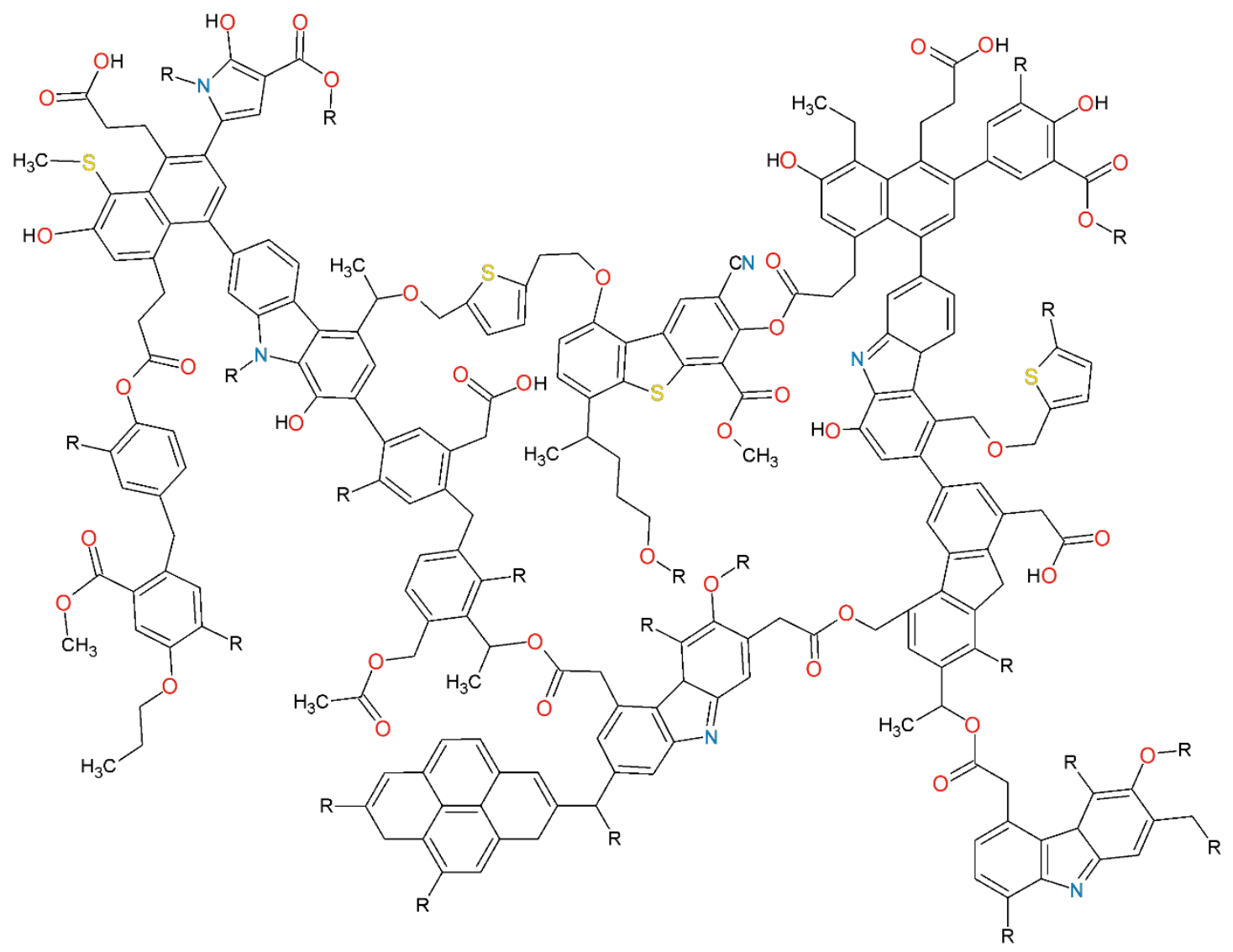

Fig. 3. Molecular model for the chemical structure of Murchison IOM. R denotes an organic moiety (Modified from Derenne and Robert $(2010)[133,134])$. This model cannot be considered as a detailed representation of the molecular structure of the IOM, but it rather illustrates the main characteristics of the $\mathrm{C}$ skeleton and the O-, N- and S-containing groups. 
Because of the low yields and poor quantification, at present Nuclear Magnetic Resonance (NMR) provides the best quantitative picture of the main functional group in the bulk IOM [135-140]. ${ }^{1} \mathrm{H}$ - and ${ }^{13} \mathrm{C}-\mathrm{NMR}$ also suggest that most of the PAUs in IOM is small and highly substituted, and that the aliphatic component be composed of short, highly branched units. There is considerable line broadening in the NMR spectra. While some of this could be due the presence of paramagnetic minerals like chromite [141], most of it certainly reflects the considerable chemical complexity. Such complexity is perhaps not too surprising given that the analyzed IOM samples are collections of very large numbers of morphologically and chemically heterogeneous grains. In addition, the NMR measurements indicate that the oxygen functionality is dominated by substituted furan/pyran moieties, highly branched oxygenated aliphatics and carbonyl groups. X-ray Absorption Near Edge Structure (XANES) spectroscopy [109,110, 142] and electron energy loss spectroscopy (EELS) [143] applied at the carbon K-edge also provides a valuable tool for assessing the functional group chemistry of IOM at scales that range down to $100 \mathrm{~nm}$ or less. C-XANES spectra exhibit a strong absorption feature corresponding to $\mathrm{C}=\mathrm{C}$ groups, likely related to aromatic rings, along with absorption corresponding to carboxylic groups. Minor absorption features due to ketone/phenol/nitrile and hydroxyl groups are also seen. As aliphatic carbon is a weak absorber at the carbon K-edge, its contribution to the spectra is faint, but noticeable.

To date, a ${ }^{15} \mathrm{~N}-\mathrm{NMR}$ spectrum has only been reported for Orgueil IOM [130]. It shows a strong contribution for pyrrole groups, along with minor contribution from nitrile. However, studies of IOM from other primitive meteorites have failed to see any ${ }^{15} \mathrm{~N}$-NMR signal above background despite having similar nitrogen contents to Orgueil IOM $[130,144]$. Nitrogen functionality can also be probed by XANES spectroscopy. The nitrogen K-edge XANES spectra are remarkably similar across chondrite groups and petrologic types, suggesting that the nitrogen functional groups are very stable. The spectra are also remarkably featureless, with only a few very small peaks despite the transitions for many nitrogen functional groups being very intense. One peak is consistent with a very small 
amount of imine and/or nitrile. Another peak is consistent with pyrrolic, amine or amide nitrogen.

Otherwise, the near continuous absorption spanning the entire N-XANES region requires the presence of a range of as yet unidentified nitrogen functional groups.

XANES also provides the best evidence for the sulfur speciation in IOM, although it has been the subject of only one study of bulk IOM [145]. In that study, the sulfur functionality was divided into three major groups with similar absorption energies: aliphatic sulfur (disulfides, thioethers and thiols), sulfur heterocycles (thiophenes and thianthrenes) and oxidized sulfur groups (sulfoxides, sulfones and sulfonates). The most intense absorption features in all the IOM spectra are due to aliphatic sulfur, with much weaker heterocycle and oxidized sulfur intensities. The relative heterocycle and oxidized sulfur intensities are higher in more aqueously altered meteorites, suggesting that there was some parent body modification of the sulfur functionality.

The Tagish Lake meteorite has a number of distinct lithologies that experienced varying extents of hydrothermal alteration, probably associated with a brief heating event(s). The IOM in these lithologies exhibit varying degrees of aromaticity (and H/C) that correlate with the extents of alteration, suggesting that the two motifs are linked $[146,147]$. The varying degrees of aromaticity seem to have been produced with little or no loss of carbon or nitrogen, although there was loss of hydrogen and isotopic evolution due to interaction with the aqueous fluid. The apparently facile and efficient conversion of aliphatic to aromatic is, perhaps, best explained if the aliphatic material in the original IOM was already organized in cyclic structures able to minimize the number of bond breaking and restructuring. This is consistent with observations in various CM chondrites of increasing alteration degrees; the IOM in more altered CMs shows more aromatic carbon, less aliphatic chains $[135,136]$ and a decreasing $\mathrm{H} / \mathrm{C}$ ratio.

The hydrogen isotopic compositions of individual pyrolysates (both aromatic and aliphatic) and aliphatic side groups on the PAUs released by $\mathrm{RuO}_{4}$ are comparable to those of the bulk IOM compositions, albeit with significant scatter [128,148,149]. It has been suggested that this scatter is the result of variations in the average hydrogen isotopic compositions of moieties in the IOM, reflecting 
heterogeneities at the molecular scale that could be correlated with $\mathrm{H}-\mathrm{C}$ bond energies [149]. This correlation has been reproduced by irradiation experiments on simple organic polymers [150]. This interpretation is not consistent with all pyrolysis results [148]. On the other hand, electron paramagnetic resonance (EPR) experiments suggest that radicals in IOM are associated with very extremely D-rich benzylic positions on aliphatic side chains $[151,152]$ that could eventually constitute localized micron-sized D-rich enrichments called hotspots [23]. The C-H bond energies of benzylic radicals is amongst the lowest in the IOM. The EPR experiments also show that the radical speciation in IOM (dominated by diradicals/diradicaloids) is quite unlike those of terrestrial coals and kerogens (exclusively monoradicals) despite their superficial resemblance in terms of elemental and functional group chemistries [153]. Irradiation by energetic particles in the ISM or solar protoplanetary disk is one possible mechanism for generating these radicals. Irradiation experiments of simple polymers have successfully produced monoradicals and diradicals but at much lower concentrations than observed in IOM [154]. Unfortunately, that study was unable to determine if diradicaloids were present after the irradiation, leaving their origin unresolved.

\subsection{Origin}

Currently, there is no consensus about where, how and from what the IOM formed [112,7]. One possibility that was originally put forward because of the large deuterium and ${ }^{15} \mathrm{~N}$ enrichments in IOM is that it or its precursors formed in the protosolar molecular cloud $[9,10,16,155,156]$. It has long been known that simple molecules synthesized in the relatively dense $\left(10^{2}-10^{6}\right.$ particles $\left./ \mathrm{cm}^{3}\right)$, cold $(10-30$ $\mathrm{K})$, radiation-rich environments of molecular clouds can become enormously enriched in deuterium relative the dominant $\mathrm{H}_{2}$ [157]. The circumstellar grains found in primitive meteorites clearly survived Solar System formation. The lifetimes of circumstellar grains in the ISM is relatively short, so an even larger amount of dust that formed in the ISM is also likely to have survived Solar System formation. The ISM silicate dust should have an average isotopic composition that is like that of the bulk Solar System, making it hard to identify. This may not be the case for carbonaceous dust. Refractory 
carbonaceous dust and large PAHs make up a significant fraction of the dust in the diffuse ISM $[158,159]$. While there are striking similarities in the aliphatic C-H stretch regions of the IR spectra of the diffuse ISM dust and IOM [160,161], the diffuse ISM dust does not seem to contain significant amounts of heteroatoms ( $\mathrm{O}, \mathrm{N}$ and $\mathrm{S})$, unlike IOM. What happens to the carbonaceous dust once it is incorporated into the cold $(10-30 \mathrm{~K})$, dense regions of molecular cloud where star-formation occurs is still a matter of debate. One possibility is that the grains would become coated in complex interstellar ices (bearing $\mathrm{H}_{2} \mathrm{O}, \mathrm{CO}, \mathrm{CO}_{2}, \mathrm{H}_{3} \mathrm{COH}, \mathrm{H}_{2} \mathrm{CO}, \mathrm{HCN}, \mathrm{NH}_{3}, \mathrm{NH}_{2} \mathrm{COH}$ etc.) and irradiated by energetic cosmic rays $[162,163]$. Radiation damage by the cosmic rays would generate defects and radicals in the grains and ices. When the grains/ices are warmed during star formation, these radicals become mobile and react adding heteroatoms to the carbonaceous grains. The $\mathrm{H}_{2} \mathrm{O}$ and many of the other compounds in the ices are known to be deuterium- and ${ }^{15} \mathrm{~N}$-rich, so this mechanism would naturally explain the isotopic composition of the IOM.

An origin in the ISM would imply that a common IOM precursor was incorporated into cometary and asteroidal parent bodies, and that in the meteorite parent bodies, at least, it was subsequently altered under a range of conditions $[10,164]$. Whether all the elemental and isotopic variations found amongst the chondrites can be attributed to parent body processes remains debated. It is possible that the IOM precursor material was modified to varying degrees in the solar nebula prior the accretion of the chondrite parent bodies $[118,165]$, but at present clear evidence that this happened is lacking. Alternatively, the IOM or its precursors could have formed in the solar nebula. Formation of IOM by FTT synthesis was the first widely accepted mechanisms for its origin $[166,167]$. However, there is no spatial association between IOM and plausible mineral catalysts (Fe,Ni-metal, magnetite, etc.). Moreover, FTT synthesis is likely to have been efficient only in a relatively narrow region of the inner Solar System [167], which would make it difficult to account for the high abundances of refractory organics in comets. In addition, experiments suggest that under nebular conditions the dominant products of FTT synthesis would be volatile gases $[168,169]$. The isotopic composition of the IOM also cannot be explained by FTT synthesis alone. 
A nebular alternative to FTT synthesis is irradiation of ices by cosmic rays and stellar radiation when they are lofted to high altitudes in the disk [170]. To date, ice irradiation experiments have not produced IOM-like material. It is possible that irradiation of organic residues after sublimation of the ice can accomplish this, but experimental evidence that this is the case is lacking. Such a nebular irradiation scenario may be rather similar to how dust is cycled between the diffuse and dense ISM, but in the solar nebula it may be harder to generate the large deuterium and ${ }^{15} \mathrm{~N}$ anomalies found in IOM. It has been suggested that the deuterium enrichments, at least, could be the result of interaction of carbonaceous grains with cosmic ray generated $\mathrm{H}_{2} \mathrm{D}^{+}[149,151]$. In molecular clouds, $\mathrm{H}_{2} \mathrm{D}^{+}$is one of the species responsible for producing highly deuterated organic molecules, but it is not clear that cosmic ray fluxes would have been sufficient to generate much $\mathrm{H}_{2} \mathrm{D}^{+}$in the solar nebula because strong stellar winds would have shielded the disk $[171,172]$.

One mechanism that has arguably been explored experimentally more than any other is polymerization of interstellar formaldehyde from solution via the formose reaction when ices melt in meteorite or cometary parent bodies $[140,173,174]$. To reduce reaction times, most experiments have included glycolaldehyde as a starting product as it is an early intermediate in the formose reaction. The process naturally produces precipitates that in size and morphology look very similar to the nanoglobules found in chondrites and IDPs. At low temperature, the product is much more oxygen-rich than IOM, but heating it briefly to $\sim 250^{\circ} \mathrm{C}$ can generate a material whose NMR spectrum resembles in many ways IOM. Despite these promising results, this mechanism must overcome significant objections. The formose reaction is relatively inefficient with yields of at best $\sim 20 \%$ and formaldehyde is a minor component of ISM and cometary ices [59]. Similar experiments conducted on hexamethylenetetramine (HMT), recently identified in the carbonaceous chondrites Murchison, Murray and Tagish Lake [175], result in the formation of a N-rich insoluble organic material but with an even smaller yield (1\%) [176]. This makes the abundances of IOM in chondrites and the related refractory organics in comets problematic. It is also unclear that the volatile-rich $\left(\mathrm{CO}, \mathrm{CO}_{2}\right.$, etc.) ices of comets like 67P/Churyumov-Gerasimenko could ever have been molten. 


\subsection{Formation pathways of putative IOM precursors and aromatic compounds}

Illustrated schematically in Fig. 4 is the standard picture of how small carbon-bearing molecules, such as acetylene, ethylene and methane, that are formed in the outflows of carbon stars can give rise to PAHs and then to carbonaceous particles through combustion-like processes $[177,178]$. However, a robust explanation of how these large molecules form is still lacking, especially in evolved stars where the characteristic aromatic infrared bands are only detected at late stages. Indeed, recent experimental work found that amorphous carbon nanograins and aliphatic carbon clusters can form through lowpressure gas-phase condensation of carbon atoms in a hydrogen atmosphere with a $\mathrm{C} / \mathrm{H}_{2}$ ratio that is similar to that reported for evolved stars, while aromatic species or fullerenes do not form effectively under these conditions [179].

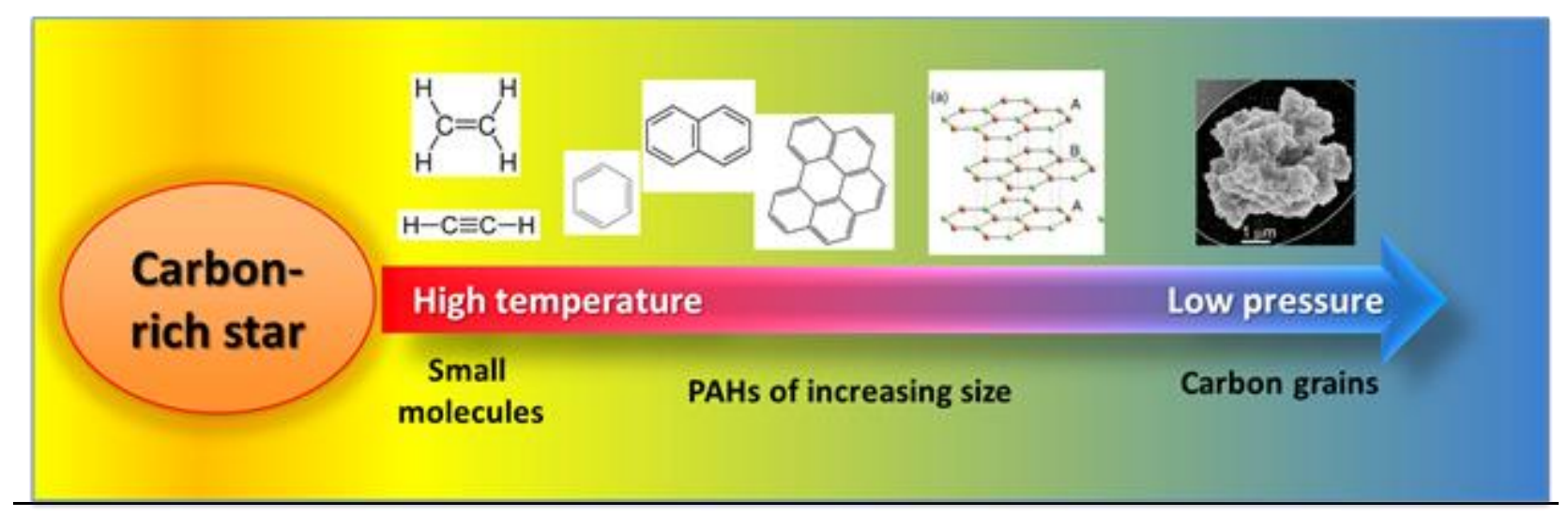

Fig. 4. Schematic representation of the formation of carbon grains in late-type carbon-rich stars.

The hydrogen-abstraction/acetylene addition (HACA) mechanism [180], involving the atomic hydrogen abstraction from benzene to phenyl radical $\left(\mathrm{C}_{6} \mathrm{H}_{5}\right)^{\circ}$ [181] followed by sequential addition of two acetylene molecules, ring-closure and aromatization [182] (Fig. 5, pathway A) has been proposed to explain the large presence of naphthalene in astrochemical environments. Recently, in a combined crossed beam and theoretical study it has been demonstrated that naphthalene can be formed in the gas phase via a barrierless and exoergic reaction between a phenyl radical and vinylacetylene. This would be a low temperature alternative to the mechanism in path $\mathrm{A}$, and challenges the traditional view that 
PAH-formation only occurs at high temperatures [183]. This mechanism has been suggested to account for the formation of naphthalene and other PAHs in Titan's atmosphere (Fig. 5, panel B) [184].

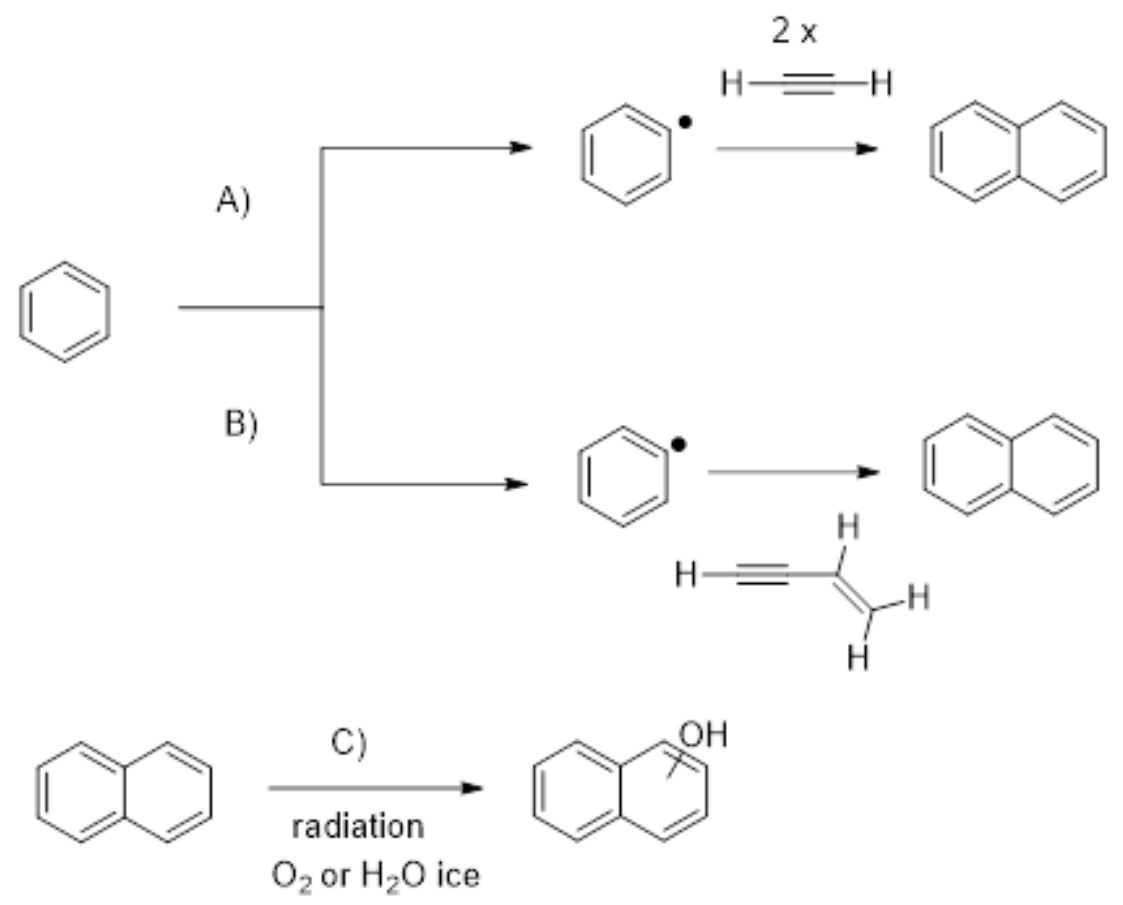

Fig. 5. Schematic representation for the radical mediated formation of naphthalene and naphthol derivatives in astrochemical environments. Pathway A: high energy addition of two acetylene molecules on a phenyl radical $\left(\mathrm{C}_{6} \mathrm{H}_{5}{ }^{\circ}\right)$ followed by ring-closure and aromatization. Pathway B: low energy alternative mechanism based on the addition of vinylacetylene. Pathway C: general oxidation pathway for the post-synthetic oxidative transformation of the naphthalene ring mediated by the formation of $\mathrm{HO}$ radicals.

Parker et al. (2012) [183] showed that the synthesis of naphthalene via this mechanism should also occur in the interstellar medium at low temperatures, including cold molecular clouds where these barrierless phenyl-type radical reactions could even lead to more complex structures like phenanthrene and anthracene [183].

The products of oxidation of PAHs, such as aromatic benzoquinone derivatives, have been initially identified in the Murchison meteorite [12]. The presence of oxidized PAHs (oxy-PAHs) in Murchison, 
and presumably in other carbonaceous chondrites, is most probably due to post-synthetic processes (Fig. 5, pathway C) [177]. Both UV irradiation and cosmic rays can induce transformation of PAHs embedded in molecular cloud ice [185,186] into a wide range of reaction products [187], including oxy-PAHs derivatives and benzoquinones [188-190]; however, UV irradiation only occurs near the surfaces of molecular clouds and does not penetrate very deeply into grains, so cosmic rays are likely to be the main source of radiation and they penetrate through the whole grains inducing probably a similar chemistry to UV in ices. These molecular cloud ices are an important potential source of the organic molecules found in primitive Solar System bodies such as meteorites, asteroids, giant planet satellites, and comets [191-193]. In particular, the oxidation of naphthalene to naphthols has been reported under a large variety of experimental conditions such as UV irradiation [194] and radiolysis [195,196]. In most of these processes, reactive $\mathrm{OH}$ radicals play important roles, performing hydrogen abstraction processes and radical coupling reactions.

Alternatively, naphthols can be produced by thermal decomposition of simple organic salt precursors of meteorite components, as in the case of the thermal decomposition of iron oxalate. In this latter case, FTT synthesis and related processes involving $\mathrm{CO}_{2}, \mathrm{CO}, \mathrm{H}_{2}$ and $\mathrm{H}_{2} \mathrm{O}$ may be responsible for the observed variety of the reaction products [196], although it is relevant to note that the CI-CM-CR chondrites are generally thought to experience temperatures of no more than $150{ }^{\circ} \mathrm{C}$. A similar reactivity has been reported in the case of thermal and photochemical processes catalyzed by clays [197], in which case naphthols can be produced from $\mathrm{CO}_{2}$ and methane by two alternative reaction pathways: i) FTT (involving methanol) and radical ring-closure to cyclohexane followed by dehydrogenation (Fig. 6, panel A); or ii) 2+2 thermal or photoinduced cyclization of ethene molecules (Fig. 6, panel B) [198]. The actual importance of photochemical processes relevant for interstellar and early solar system processes needs however to be assessed, since photochemistry is only important at the very surface of an asteroid or comet, for instance, and only a tiny fraction of their masses can be processed in this way. Overall, these lines of evidence would indicate that the aromatic precursors of melanin, including 
naphthalenes that were available in the various presolar astrochemical environments probably acted as reagents for polymerization processes that could ultimately have contributed to the formation of IOM.

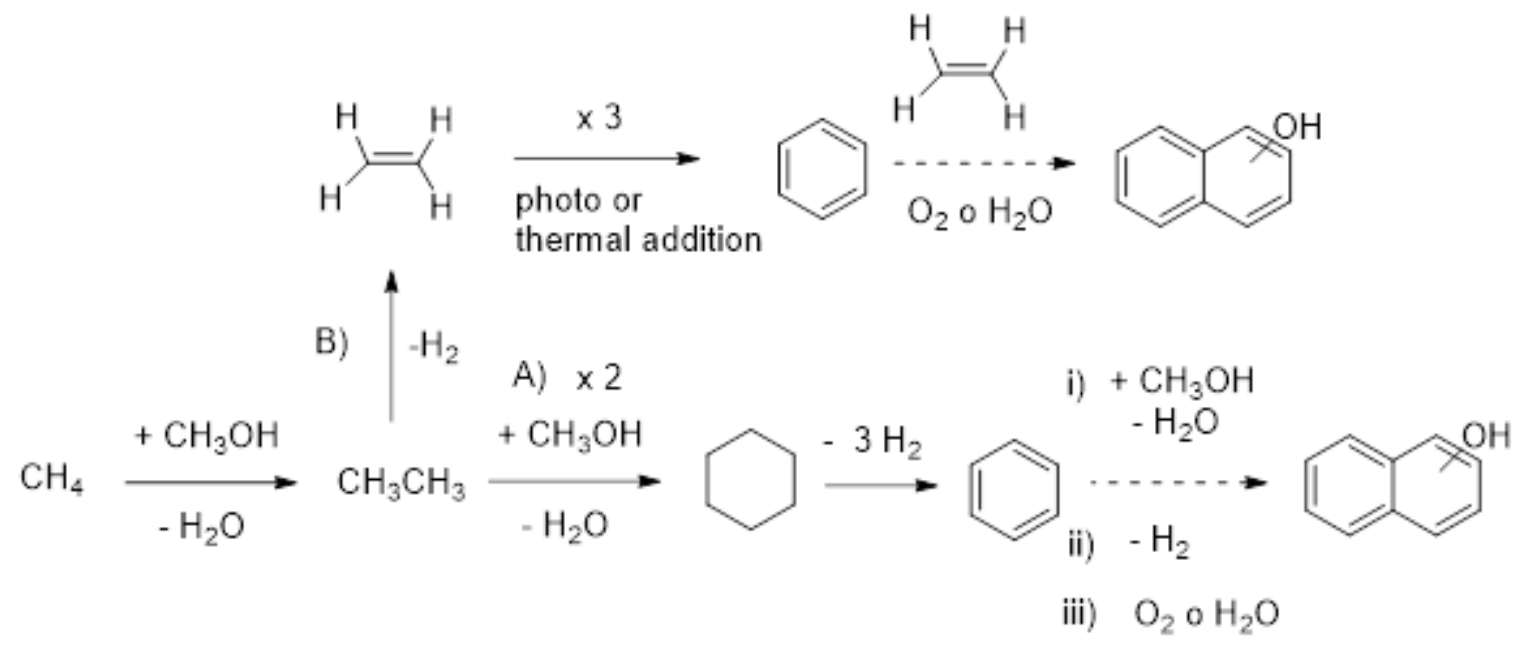

Fig. 6. Formation pathways of oxy-PHAs. A: Sequential FTT process involving methane and methanol followed by radical ring-closure dehydrogenation and terminal oxidation. B: FTT process involving methane and methanol to yield ethene followed by sequential thermal or UV cyclization and final oxidation.

In support of this view, TEM observations of carbonaceous chondrite acid residues indicated at least two morphologically distinct components of the IOM $[199,200]$ - the major component has a fluffy texture, while the minor component occurs as solid or hollow globules/nanoglobules (also sometimes referred to as nanospheres) (Fig. 7). The fluffy material in Orgueil IOM is largely amorphous [199] but contains some regions with fringe spacings of $0.21 \mathrm{~nm}$ and $0.34-0.39 \mathrm{~nm}$, roughly corresponding to the (100) and (002) d-spacings of graphite, respectively. Derenne et al. (2005) [201] interpreted the fringes as being local regions of order due to stacking of 2-3 of layers PAHs that are typically 2-3 rings across (i.e., 4-9 rings/layer) [201]. However, others suggest that the fringes are the result of radiation damage in the TEM $[6,143]$. 

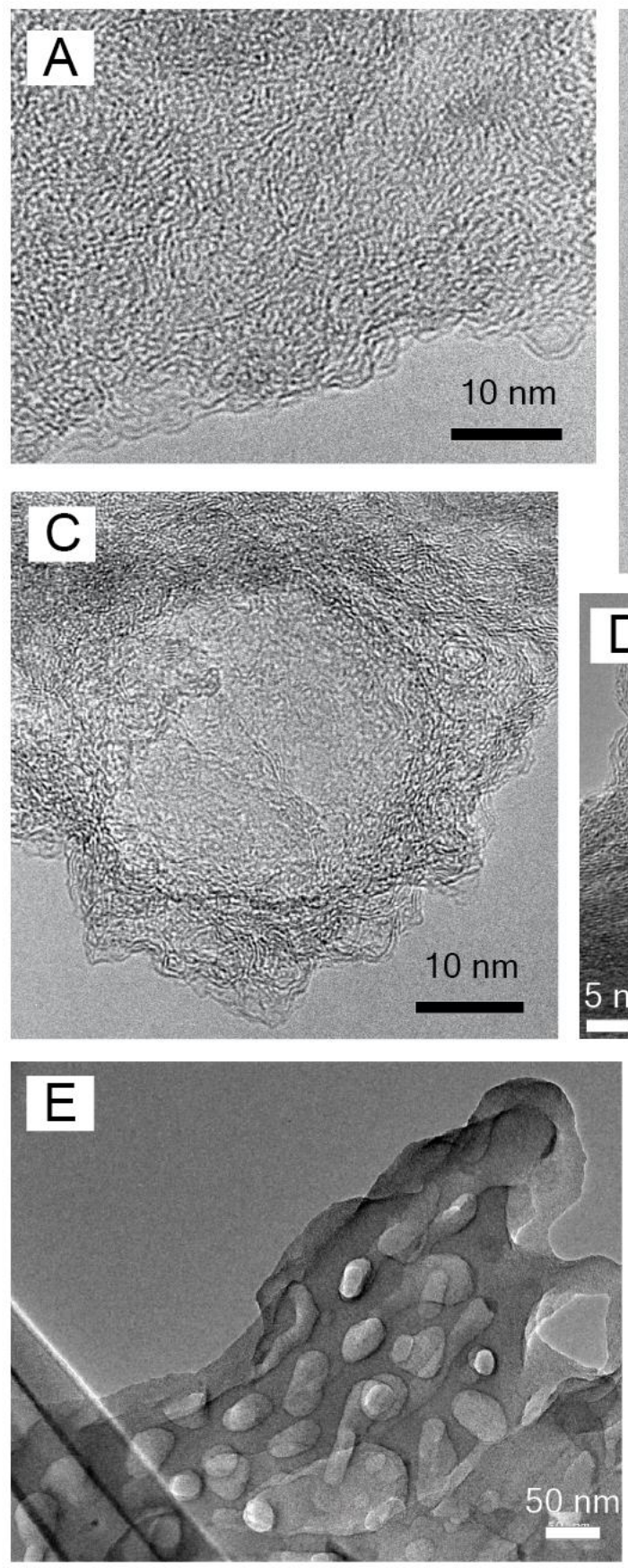
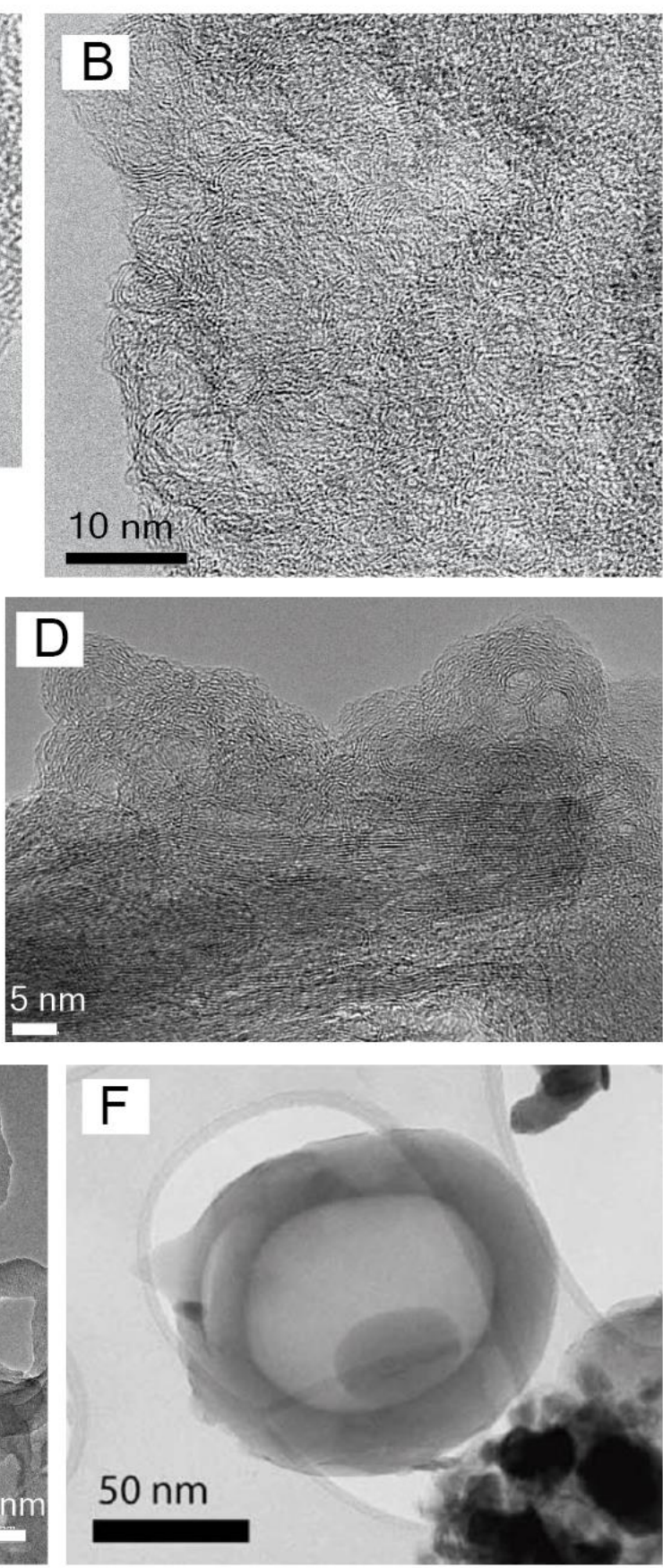

Fig. 7. TEM images revealing the various textures exhibited by IOM in chondrites A: Murchison CM2, B and C: Kainsaz CO3, D and E: Abee EH4, F: Tagish Lake. IOM usually displays a disorganized structure but can present significant porosity that can form holes and vacuoles (see C, D and E). In several type 1 and 2 carbonaceous chondrites, IOM can exhibit nanoglobules (F) which origin remains debated. Images from Le Guillou et al. (2012) [200] (A, B and C), Remusat et al. (2012) [165] (D and E) and Garvie and Buseck (2004) [134] (F). 
Besides the forementioned processes in the nebula or molecular clouds, insights integrating and/or supporting current views about the role of PAHs in prebiotic processes can be gained by looking at denser environments like Titan's atmosphere, which is considered a good proxy of our primitive Earth's atmosphere. Titan, the largest moon of Saturn, has long been a subject of interest because it provides an excellent example of abiotic processing of organic material. Titan's atmosphere contains nitrogen, as major constituent, and unsaturated hydrocarbons, like acetylene, ethylene and butadiene. These are key species for the formation of PAHs and nitrogen-containing PAHs (NPAHs), the latter being important prebiotic species. In fact, they can be considered the missing link between N-bearing acyclic molecules and prebiological systems, like nucleobases. Recently, Parker and Kaiser reviewed different reaction pathways leading to the gas-phase formation of NPAHs via radical-mediated aromatization reactions [202]. For example, pyridine can be formed from the reaction of vinyl cyanide and its radical or from cyano radicals reacting with 1,3-butadiene. Furthermore, Parker and Kaiser experimentally demonstrated the feasibility and reliability of aromatization reactions occurring via neutral-neutral interactions, thus explaining how chemical evolution can proceed from acyclic systems to polycyclic aromatic hydrocarbons and showing that these are barrierless reactions that are thus feasible at low temperatures [202]. The Ion Neutral Mass Spectrometer (INMS) and the Cassini Plasma Spectrometer (CAPS) instruments onboard the Cassini spacecraft discovered the presence of a large variety of carbocations and carbanions, ranging in size from small species to heavy positive (up to $350 \mathrm{amu}$ ) and negative (up to 10,000 amu) ions [203]. In Ali et al. (2015) [203], the composition detected by the Cassini mass spectrometers was investigated in terms of molecular structures and the formation of complex macromolecules explained in terms of reaction mechanisms from simple molecules to complex polycyclic hydrocarbons, thus demonstrating how PAHs and NPAHs grow in size not only via neutral-neutral reactions, but also via ion-neutral reactions. Subsequently, the Nobel laureate G.A. Olah [204] extended Titan's carbocation chemistry of Ali et al. [203] to the interstellar one, thus showing a clear link between Titan's atmosphere and the interstellar medium and thus between their chemistries. 
In summary, there is a lot of debate about the origin of PAHs and aromatic compounds in general. Cstars are certainly a/the major source of carbonaceous dust being added to the ISM but little is known as to how much of the dust in the ISM formed around C-stars. The current belief is that dust lifetimes in the ISM are short compared to residence times, and most dust/PAHs must form in the ISM/MCs, but how this happens is unclear.

\section{Melanins as evolutionarily preserved complex multifunctional organic polymers}

\subsection{Classification, occurrence and physicochemical properties}

Production of melanin is a universal and at the same time enigmatic form of adaptation of living organisms to the variable conditions of the Earth. The presence of various kinds of melanins in representatives of almost every large taxon suggests the evolutionary importance of melanogenesis. The traditional classification of melanins divides them into the nitrogen-containing eumelanins, pheomelanins and neuromelanins in animals, all derived from the amino acid tyrosine, and the nonnitrogenous allomelanins and pyomelanin found in plants, fungi and lower organisms that are derived mainly from a range of hydroxylated aromatic precursors [205].

From an evolutionary perspective, the appearance of nitrogen-containing units in melanin pigments would thus seem to mark the passage from unicellular or lower organisms, including fungi and plants, to the animal kingdom. At a finer level of analysis, the presence of a mutated phenotype with reddish sulfur-containing cysteine-derived melanin pigments, the pheomelanins, seems to be associated with the higher levels of evolution, including birds, mammals and humans (the red-haired Celtic phenotype). For the purposes of this paper, the main focus will be on the properties of those melanins found at the lowest evolutionary levels that may be closer to the prebiotic precursors, especially those in bacteria and fungi. For exhaustive coverage of the topic the reader is referred to several recent reviews [206-209].

Synthesis of melanins in bacteria was initially thought to occur only through the DOPA pathway. In the seventies a brown pigment derived from homogentisic acid was identified in Pseudomonas 
aeruginosa and was referred to as pyomelanin (Fig. 8) [210]. The same type of pigment was later identified in Streptomyces avermitilis [211] and again in Pseudomonas putida [212,213].

Homogentisate-derived melanin formation was described in mutated Vibrio cholerae strains [214] and in mutated Burkholderia cepacia complex isolates [215].

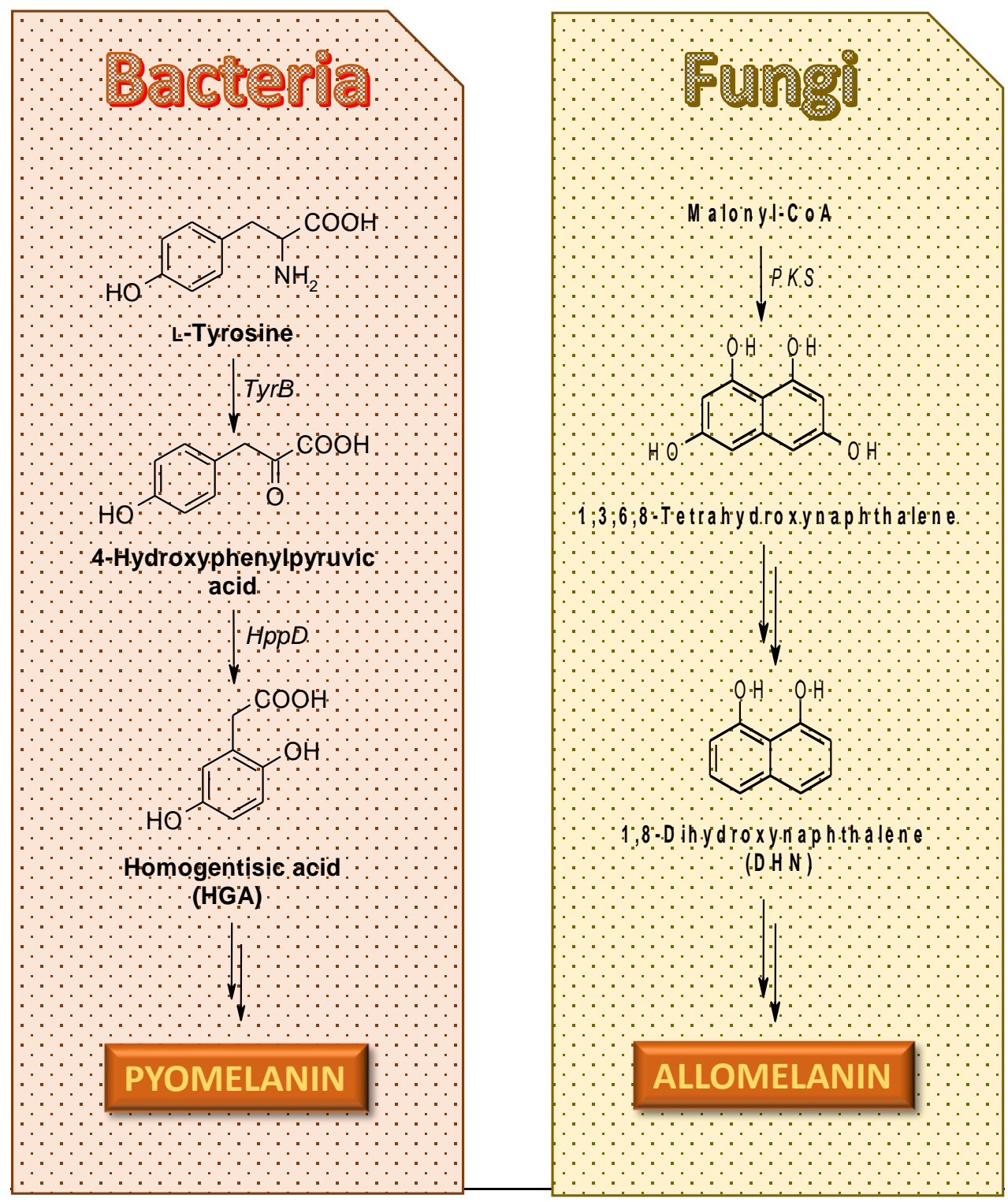

Fig. 8. Schematic view of the main melanin biosynthetic pathways in bacteria and fungi. Minor pathways have been omitted for the sake of simplicity.

In fungi, melanins arise mainly via two pathways (Fig. 8) [208]. The main one stems from the oxidative polymerization of 1,8-dihydroxynaphthalene (DHN), a metabolite produced via a polyketide synthase (PKS) pathway [216-219]. The polymerization process may be brought about by multicopper 
oxidases in Asperigillus fumigatus [220,221], Cochliobolus heterostrophus [222] and Cochliobolus orbiculare [223]. A few fungi synthesize melanins starting from tyrosine, via the oxidative polymerization of its metabolites DOPA and homogentisic acid, this latter leading to the formation of the brownish pyomelanins [224,225].

Herein, for the sake of simplicity, all non-nitrogenous melanins from bacteria and fungi will be collectively referred to as allomelanins. Elemental composition data for representative examples of bacterial and fungal melanins is reported in Table 2.

Table 2. Comparative view of elemental compositions of bacterial and fungal melanins
$\mathrm{C}(\%) \quad \mathrm{H}(\%) \quad \mathrm{O}(\%) \quad \mathrm{N}(\%) \quad \mathrm{S}(\%)$
Reference

\section{Fungal melanin}

Cryptococcus

58.99

7.11

28.34

5.56

[226]

neoformans

Spissiomyces

52.69

4.69

$42.15 \quad 0.47$

endophytica

Tuber melanosporum

47.2

6.0

45.2

1.0

$<0.3$

Auricularia auricula

41.18

5.56

51.60

1.66

Termitomyces

54.68

3.54

$26.92 \quad 2.49$

12.36

albuminosus

Tuber melanosporum

52.10

4.20

28.31

6.16

9.23

Vitt.

Boletus griseus

56.38

5.86

28.04

6.17

2.44

\section{Bacterial melanin}

\begin{tabular}{lcccccc}
\hline Klebsiella sp. GSK & 47.9 & 6.9 & - & 12.0 & 0.9 & [232] \\
Bacillus subtilis & 70.0 & - & - & 12.0 & 4.1 & [233]
\end{tabular}


$4 N P-B L$

Studies over the last decades have clarified some important chemical and physical properties of allomelanins [207] that are common to all members of this class of polymers regardless of structural and biosynthetic differences [235]. These properties include, among others: $a$ ) a black or dark coloration due to broadband, often featureless, absorption spectra covering the entire UV-visible range [236]; b) a marked structural heterogeneity; c) almost complete insolubility in water and organic solvents; $d$ ) an intrinsic paramagnetic character responsible for a broad stable EPR signal [237]; $e$ ) semiconductor-type electrical conductivity properties [238]; $f$ ) efficient non-radiative excited state deactivation; $g$ ) a redox behavior associated with antioxidant activity; $h$ ) a strong metal cation binding and drug affinity, and $i$ ) efficient gas adsorption properties.

Most of these properties, especially a) and d-g), are evidently associated with the unusual chemical complexity of all melanin-type polymers at the level of the conjugated $\pi$-system. Such a complexity stems from various interrelated levels of structural disorder, including monomer disorder, molecular weight disorder, isomerism-related disorder, redox disorder and supramolecular disorder [236,237].

\subsection{Biological roles and functions}

The ability to produce melanin from various phenolic substrates is typical of various microorganisms; however, although the subject of multidisciplinary investigations since time immemorial, the biological roles of melanins are still a matter of debate. Besides photoprotection, the accepted functions of most melanins include camouflage, antioxidant and free radical scavenger defense, UV dissipation, ionic conductor behavior, and de-toxification via binding of metals and xenobiotics [239]. These diverse functions suggest that the utility of melanogenesis is rooted in the peculiar physical and chemical characteristics of the pigments. Indications of the past biological role of melanin pigments can be obtained from the occurrence of allomelanins in several bacteria and fungi that appeared on Earth before plants and higher organisms. Black melanin pigments in bacteria play diverse biological 
roles, including protection from UV radiation, heavy metals and oxidative stress, and have been implicated in pathogenesis and survival in adverse environments (Fig. 9) [206].

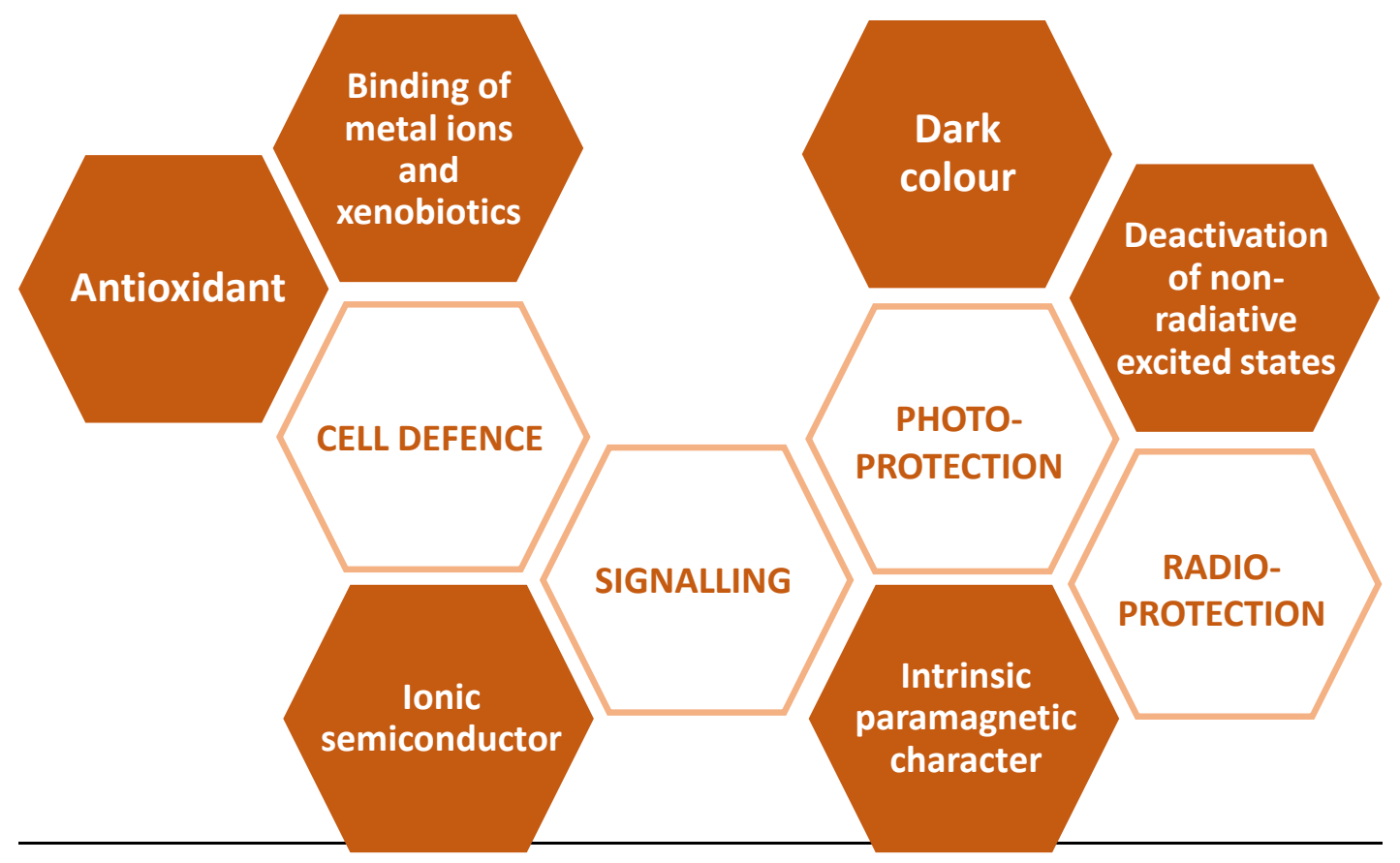

Fig.9. The conceptual interconnections between main properties (full hexagons) and biological functions (empty hexagons) of melanins.

In Bacillus anthracis, pigmented mutants are more resistant to UV exposure [240], and melanized spores of Streptomyces griseus are more resistant to UV irradiation than colorless mutants [241]. In addition, melanized Pseudomonas aeruginosa strains are more tolerant to oxidative stress [242]. Interestingly, protection of bacterial melanin from photodamage can be extended to the organisms living in close association, as in the case of the darkly pigmented sponges that contain Vibrio, Providencia, and Shewanella symbionts [243]. The important role of melanins is supported by the fact that several non-phylogenetically related bacteria sharing the same ecological environment produce the pigment.

In fungi melanin is an important protective factor against the adverse effects of environmental stresses, such as UV radiation, heat, cold, drying, salts, heavy metals and radionuclides [244]. Melanins are believed to account for the ability of fungi to survive high levels of ionizing radiation [245,246]. Under 
extreme conditions, mycobiota usually produce higher proportion of melanized fungi, such as in the case of radionuclide contamination. Likewise, the amount of melanized spores is higher in air exposed environments than in soils and dark-colored spores are more resistant to UV irradiation. Microcolonial yeast-like fungi that grow on rocks under the conditions of extreme temperatures, e.g. in the Antarctic, or drought and low concentrations of organic compounds, are strongly melanized. On this basis, it can be concluded that melanin production in fungi represents an example for evolutionary convergence, being produced at the end of different metabolic pathways, even in one taxon, as a means of achieving efficient adaptation. The possible evolutionary origins of melanogenesis have been suggested to include extracellular oxidative polymerization of phenolic compounds caused by enzymes supporting this process, followed by a gradual adaptation to the intracellular control of the process.

The question of melanin's radiation protective function can be also looked at from a paleobiological perspective [209]. Highly melanized fungal spores have been detected in deposits of the early Cretaceous period when the Earth crossed 'magnetic zero' with decreased defense against solar and stellar radiation [247]. In this scenario, the dying out of many species of animals and plants was hypothesized to involve an unseen solar companion star, a hypothetical red dwarf or brown dwarf generally referred to as Nemesis, triggering periodic mass extinction, although this hypothesis has been questioned [248]. Melanin-related virulence may have also been implicated in the possible proliferation of fungal species that contributed to the extinctions at the end of the Cretaceous period [249]. In concluding this section, we concur with Dadachova et al., 2007 [209] that: "Melanins are ancient pigments that are likely to have been selected in evolution because they enhanced the survival of microbes in both the environment and in various hosts. The emergence of melanin as a nonspecific radioprotective material may be a result of the relative ease with which these complicated aromatic structures can be synthesized from a great variety of precursors."

5. The role of IOM in prebiotic processes: from the melanin connection to the PriME scenario.

\subsection{Structural and physicochemical similarities between IOM and allomelanin}


As mentioned previously, a point-by-point comparison between the IOM and allomelanin should provide the reader with the proper conceptual basis to support the PriME scenario. However, before addressing this issue it seems necessary to carefully consider the following caveats:

1) it may sound incongruous to compare petrified organic mixtures from different chondritic samples, that experienced a variety of astrophysical conditions, with the products of genetically- and enzymatically controlled biosynthetic pathways like the allomelanins.

2) a direct comparison of elemental compositions and other structural data is likewise difficult within the melanin group, considering: a) the presence in natural pigments of the biological matrix possibly affecting most analytical data, b) the broad variety of purification protocols for natural pigments, making reproducibility an arduous task (use of synthetic melanins is generally preferred for comparative purposes), and c) the wide range of melanin pigments with different compositions, which would make an a priori choice of the reference group both questionable and arbitrary.

Bearing in mind these caveats, it is noteworthy from the preceding overview that chondritic IOM shares fundamental physico-chemical properties with allomelanin-type materials. More generally, it appears that structurally disordered organic polymers from phenolic precursors share a number of properties in common that partially override their intrinsic (bio)synthetically-determined structural diversity. These properties, or more properly, emergent properties, reflect strong intermolecular interactions between the highly heterogeneous molecular components that determine basic aggregation processes underlying the amorphous morphologies of most of these polymers and eventually account for their complex natures and exotic features. Evidence in support of the analogy between IOM and allomelanins comes mainly from elemental composition data, solid state ${ }^{13} \mathrm{C}$ NMR, EPR and X-ray spectroscopy, and TEM analysis.

A comparison of the EPR signal features of IOM from Murchison, Orgueil, Paris, Tagish lake (g-factor values centered around 2.0031) and of allomelanins from Aspergillus niger and Aspergillus fumigatus (g-factor values of 2.0035) would suggest similar carbon-centered radicals. This observation 
corroborates the view that strong aggregation and intermolecular perturbation of $\pi$-electron systems in the solid state generates chemical complexity that can produce emergent properties from self-assembly and self-organization as universal features of primitive disordered organic polymers. An overview of the unifying set of properties and characteristics of IOM and allomelanins is given in Fig. 10.

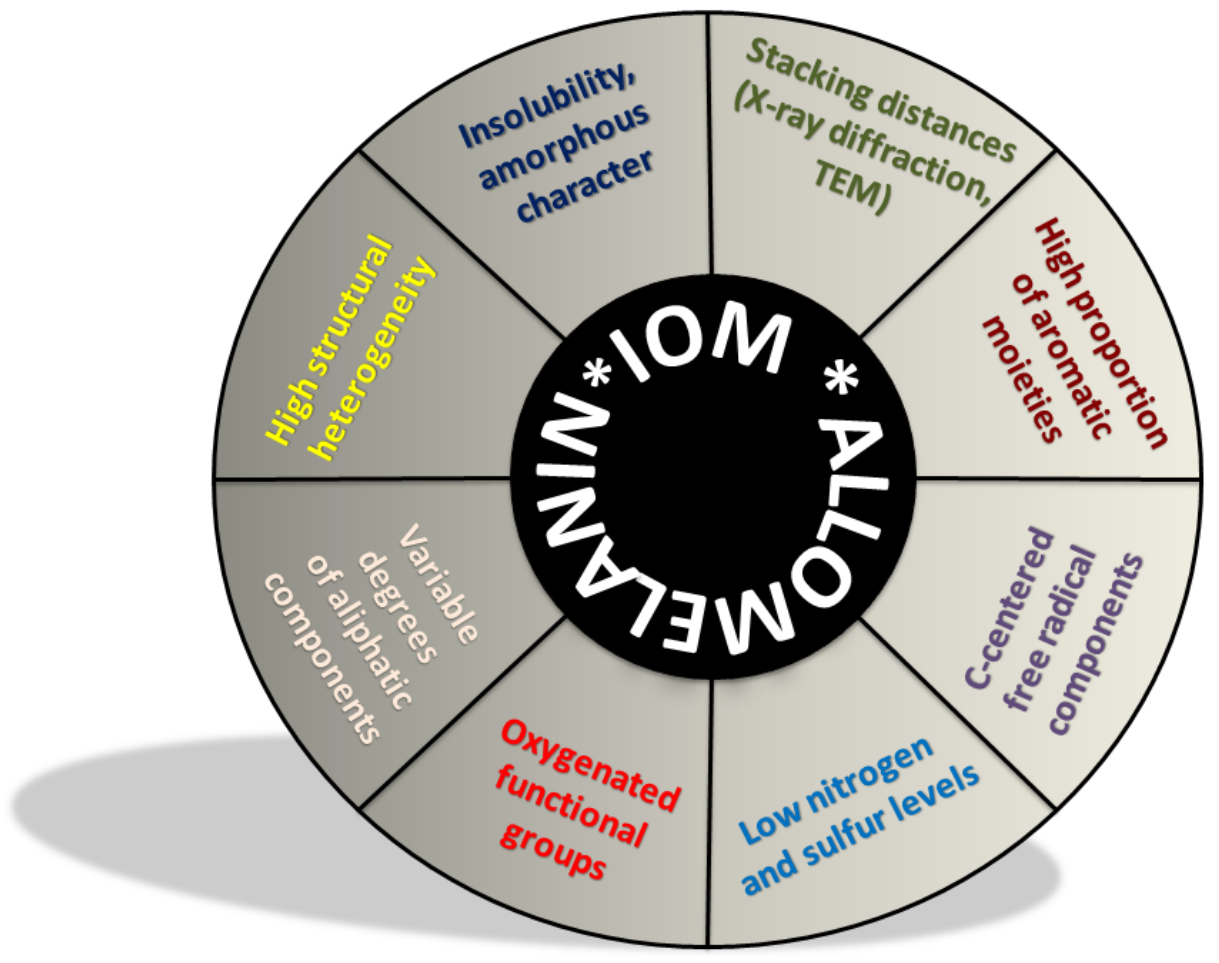

Fig. 10. Unifying set of properties and characteristics of IOM and allomelanins.

The above similarities, coupled with the known properties and functions of allomelanins and most other complex phenolic polymers, provide the basic chemical and physical ground for inferring the possible role of IOM in abiogenesis and the origin of important prebiotic molecules.

\subsection{Chemical requirements at the origin of life}

The origin of life and of life-related molecules, be it on the Earth or other planetary or non-planetary contexts, has been suggested to depend on at least eight critical reaction conditions: (1) reductive gas phase, (2) alkaline $\mathrm{pH},(3)$ freezing temperature, (4) water, (5) dry/dry-wet cycle, (6) coupling with 
high energy reactions, (7) heating-cooling cycle in water, and (8) input of life building blocks from, e.g., carbonaceous chondrites [250], comets [102], and micrometeorites [251].

Connected with these reaction conditions are three different sets of basic requirements that are schematically illustrated in Fig. 11.

\section{Advanced \\ chemical \\ requirements}

Compartmentalization

Synthesis and conversion

Replication mechanisms

Basic chemical requirements

Diversity of building blocks

Robust and specific catalysts

Energy transducers and redox mediators

Templation and product stabilization

\section{Basic physical and environmental requirements}

\section{A far-from-equilibrium system with adequate energy sources Non-destructive conditions and a suitable geological context}

Fig. 11. Main physical and chemical requirements for the origin of life

A reductionistic and unifying scenario that could fulfill most of these essential requirements for the origin of life and the need for efficient energy dissipation must include a number of requisites such as: a) provision of all the elements and essential life molecules building blocks; b) a versatile catalytic ability; c) efficient energy transducing properties in a far-from-equilibrium system; d) redox behavior; e) distinct aromatic domains providing templating motifs for biomolecule, e.g., nucleobase or porphyrin, self-assembly; f) the capacity to protect life-molecules and evolving protocells from damaging radiation and other destructive agents; g) the ability to ensure efficient compartmentalization 
for the assembly of protocells, minimal cells and other microenvironments supportive of life; h) facile replication mechanisms.

Based on the data reported in the present review, it is reasonable to hypothesize that IOM, by virtue of its similarities with allomelanins may have served most of the functions expected for the PriME in abiogenesis, as briefly summarized below.

a) IOM, like all melanins and phenolic polymers, incorporates, besides carbon, various oxygenated, nitrogenous and sulfur-based groups that may have produced small, reactive molecules upon UV, chemical or thermal degradation that were useful in the development of early life [252-254].

b) IOM, by virtue of its structural similarity with melanin-type polymers, could have provided a catalytic environment for various processes (an example of catalytic properties of melanins is provided in [255]).

c) IOM may have played a role in energy transducing mechanisms (see e.g., melanin role in organic electrochemical transistor (OECT) experiments [256]). In this regard, IOM first and melanin later might have been crucial players in Michaelian's (2017) thermodynamic dissipation perspective [257]. In this latter, the biotic-abiotic co-evolution of the biosphere was envisaged as being based on the observation that the photon dissipation efficacy of Earth in its solar environment has increased ever since the formation of Earth in the Hadean and that this evolution has incurred the dissipative structuring of pigments: “...Their proliferation to far beyond equilibrium concentrations can be understood in terms of non-linear, non-equilibrium thermodynamic principles directing autocatalytic photochemical reactions in which these pigments catalyze the dissipation of the same thermodynamic potential (the solar photon flux) that produced them."

d) IOM can serve as a protective support for biomolecule assembly, preventing their photodegradation. Indirect evidence supporting this hypothesis comes from the known quenching ability of melanins toward photoexcited states [258]. 
e) IOM can be implicated in very primitive and non-classic compartmentalization and replication processes at the origin of life. For example, TEM observations [200] revealed small cavities formed by defects in aromatic plane stacking that may be implicated in compartmentalization phenomena and specific confinement of chemical transformations. A relevant observation suggesting possible control over the interplay of metabolic and replication processes relates to the ability of non-polar molecules extracted from the Murchison carbonaceous chondrite to form vesicles structure. Reactions in liquid crystalline organizing matrices have also been proposed as a promising approach for the way primitive cells could have emerged on the early Earth [259].

\subsection{The PriME scenario and the Universal Multifunctional Adaptive Mediator (UMAM) paradigm.}

The preceding sections identified three main pillars for the "Primordial Multifunctional Organic Entities -PriMEs" scenario: a) IOM can be regarded as an archetypal abiotic melanin sharing key properties in common with fungal melanins and other forms of primitive organic matter, like kerogens; b) melanins are evolved, specialized and genetically-determined biopolymers that play a broad variety of roles in an adaptive and evolutionarily-determined manner; and (by implication): c) IOM from chondrites and other sources may provide a missing universal multifunctional mediator in the early Earth because of its melanin-like properties, whereby it can fulfill, or contribute to fulfill, most of the chemical requirements for the transition from an inorganic world to a prebiotic world producing structurally defined biomolecules.

In this scenario, IOM may well be a source of PriMEs operating at the crossroads of various prebiotic processes, because of:

a) a hybrid robust structure deeply incorporated into the stony matrix following extensive thermal processing; 
b) a broad range of chemically diverse functional groups and life-relevant elements, from nitrogen to oxygen and sulfur;

c) possible melanin-type physicochemical properties, including energy absorption, transduction and dissipation, a free radical character, catalytic activity, high adsorption capacity for gases and organic substances, metal chelation [260]

The striking analogy of IOM with melanin biopolymers, especially allomelanins from lower organisms, may be finally integrated into the novel paradigm of the Universal Multifunctional Adaptive Mediator (UMAM, see Fig. 12) that has been preserved and transformed through the development of the biosynthetic machinery for melanins to fulfill the manifold requirements for abiogenesis. From this perspective, melanins can be considered as a genetically/biochemically controlled evolution of the IOM/PriME.

\section{The Background} A PrimE could have played
important roles in prebiotic

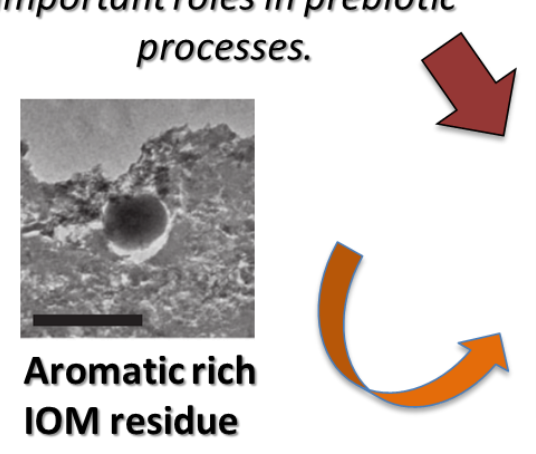

\section{The Clue}

IOM shows many properties in common with bacterial and fungal allomelanins

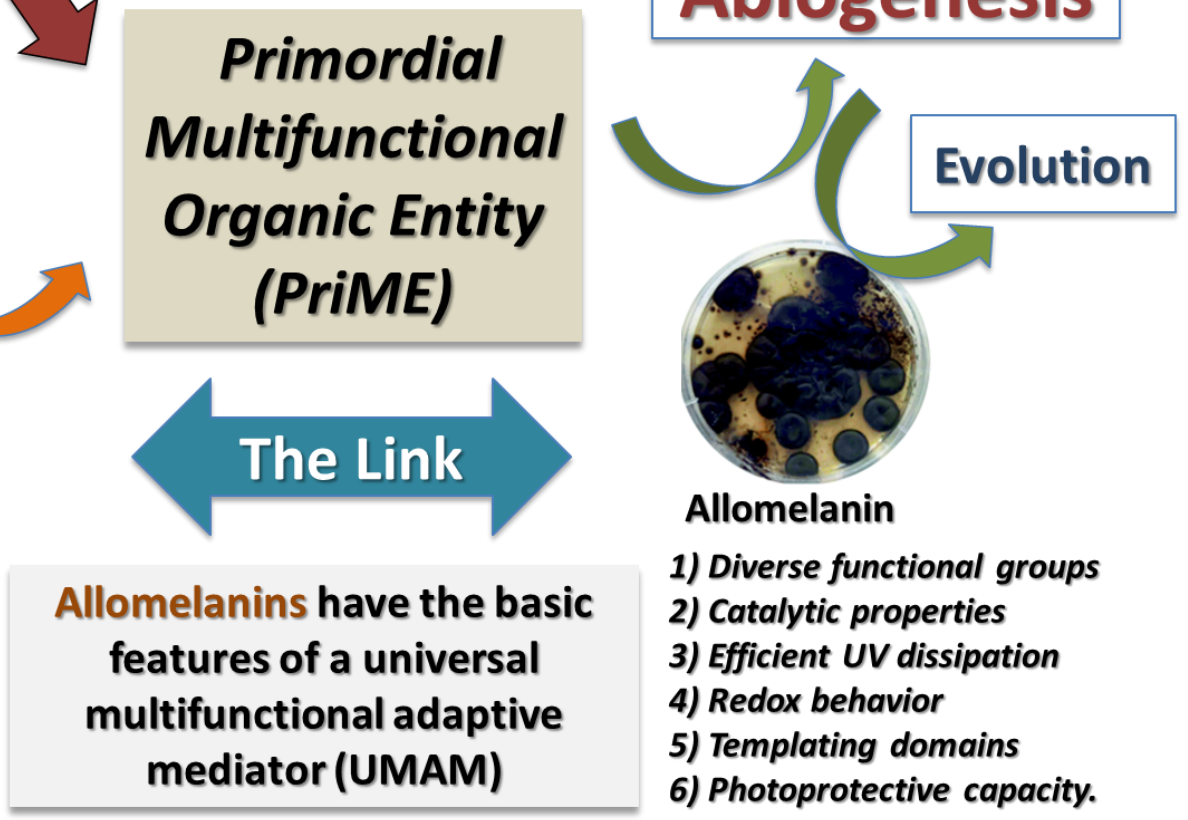

\section{The Scenario}

IOM could have served as PriME providing a universal basis for the key requisites of life: compartmentalization, metabolism and replication.
Allomelanin
2) Catalytic properties
3) Efficient UV dissipation
Redox behavior
6) Photoprotective capacity.

Fig. 12. The conceptual background of the PriME scenario and the UMAM paradigm. 
Some of the most stimulating challenges that should be addressed in ongoing and future studies include: a) defining in more detail the structure and origin of IOM, through the use of synthetic models, b) probing the potential role of IOM as prebiotic mediator via a solid experimental approach, and integrating the emerging findings about IOM into the most advanced models and theories about the origin of life. Important issues in this connection include:

1) the mechanisms of conversion of IOM into small building blocks that would be useful in the development of life, a process that is probably related to the exposure of chondrites to energetic radiation, discharges, thermal shocks and/or aqueous environments at the surface of young planets;

2) the demonstration that the organisms closer to the roots of the tree of life use melanins, for instance, the organisms that were responsible for 3-3.5 billion years old stromatolites or for the rise of $\mathrm{O}$ at 2.5 billion years.

\subsection{The PriME scenario and Virtual Reality}

A promising approach to inquire into the role of PAHs in the formation of IOM and as both a template and a mediator in key prebiotic processes would be based on advanced computational tools like artificial intelligence (AI) and immersive virtual reality (IVR) coupled to powerful hardware and software for numerical simulations. A new perspective is offered by the transition from the macroscopic to the microscopic world within a virtual reality framework thanks to the extension of a very general immersive IVR tool (Unity-3D [261]) to the molecular world (Unity-Mol [262]) and the development of commodity hardware with remarkable performance (e.g., Vive [263] or Oculus Rift [264]). In particular, a general tool, named Tardis, was developed, which can be employed for the exploration of chemical events occurring in the interstellar space, on exoplanets or meteorites and on the primordial Earth [265-267]. 3D representations of putative aromatic building blocks of IOM and of fungal melanin (Fig. 13) may be useful in this regard as a basis to enquire into the physical and chemical properties of these materials once properly framed into a IVR prebiotic scenario. 


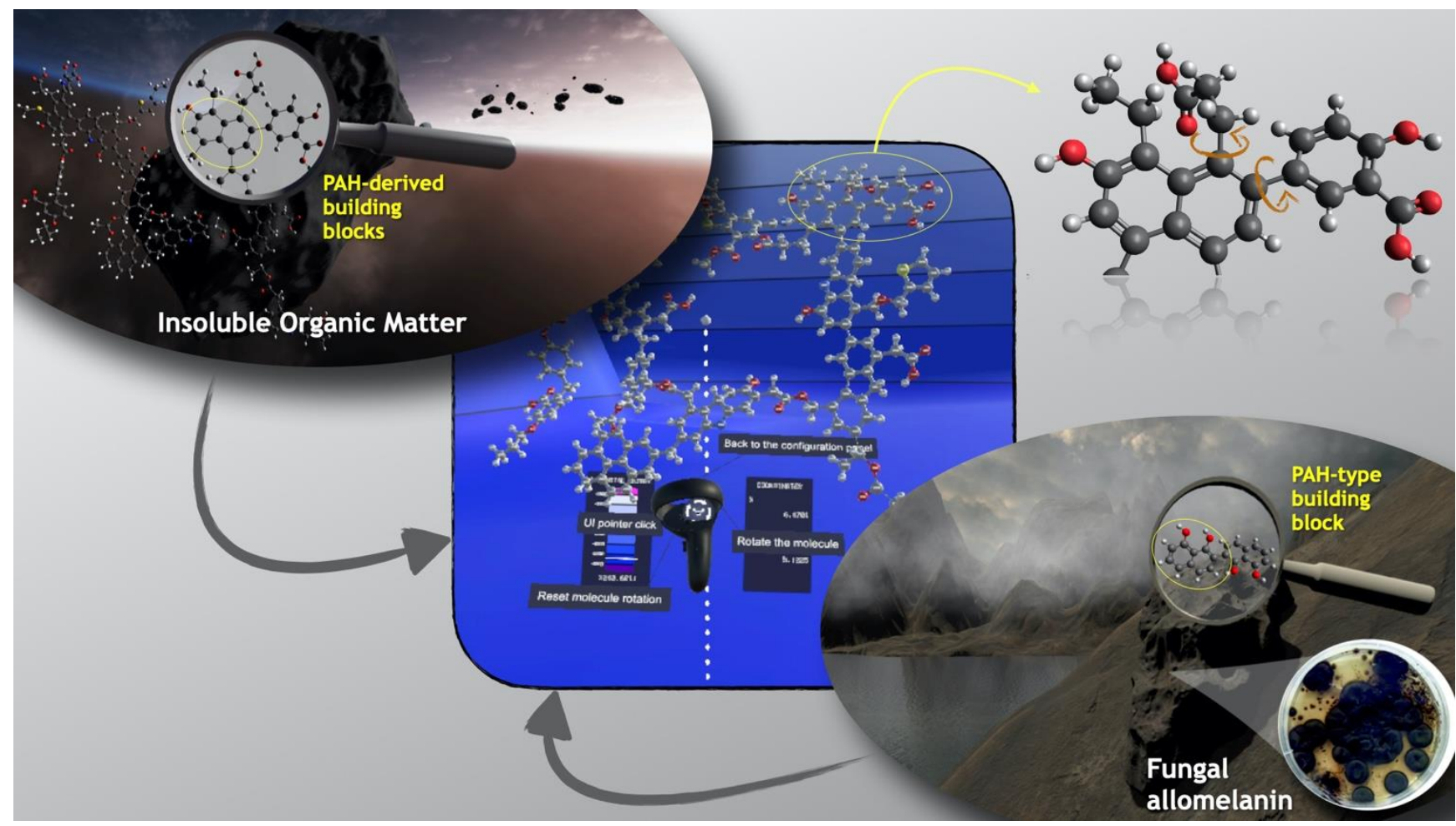

Fig. 13. Transition from the macroscopic to the molecular world in two representative scenarios: PAHderived building blocks on a carbonaceous meteorite and on fungi on the Earth. After the molecular system is perceived, two torsional degrees of freedom have been selected and the corresponding PES generated and analyzed in an IVR context.

Virtual reality may indeed allow inspection of a specific asteroid by opening the metadata associated to it, then shifting to the microscopic level, where the structure and properties of the minerals composing the asteroid and, possibly, a chemisorbed molecule like a PAH, can be investigated. As an alternative scenario, reactions involving PAHs can be visualized and investigated in the virtual environment of an exoplanet, where hypothetical fungi could evolve and produce, inter alia, allomelanins. Then, starting from an automatic perception of the molecular system, it is possible to analyze structural and conformational properties thanks to the effective integration of AI and IVR tools. For instance, by properly reducing the dimensionality of the potential energy surface (PES) to two coordinates and building a three-dimensional representation where the selected coordinates are used as the latitude and the longitude and the associated value of the PES as the elevation, it is possible 
to explore the conformational behavior (see Fig. 13) or the reactivity of the target system or of its fragments by an immersive exploration, in which the user can walk around a virtual world having the PES as terrain.

It is expected that new exciting opportunities can derive from the latest developments of immersive virtual reality approaches as versatile tools for aiding multidisciplinary research like those at the origin of life.

\section{Conclusions and perspectives}

The present survey developed from the realization that the IOM from chondrites and the allomelanins found in lower organisms display similar physicochemical properties. Considering that: $a$ ) spectral evidence suggests the presence of PAH-related aromatic domains in chondritic IOM; $b$ ) PAHs and related aromatic species are believed to be some of the primary components of complex organic molecules in the interstellar medium; c) PAH-type systems, like DHN, are the key building blocks of allomelanins, and $d$ ) allomelanins, like DHN melanin, exhibit physical features and chemical properties that may be compatible with a role in primitive compartmentalization, catalysis and replication phenomena, it can be argued that IOM delivered in favorable environments by chondrites may have played a role as a PriME, promoting key prebiotic processes by means of its PAH-derived composition.

The PriME scenario and other commonly accepted prebiotic scenarios are not mutually exclusive. It is therefore important to test prebiotic models based on the PriME scenario by addressing a number of pending issues, including in particular the following:

1) the occurrence, origin and role of PAHs in IOM from chondrites;

2) the ability of IOM from chondrites to behave as multifunctional allomelanin-like systems playing key roles in putative prebiotic processes; 
3) the processes underlying the postulated transition from chemically-controlled PAH polymerization in IOM and related materials delivered to the early Earth to the genetically and enzymatically-controlled allomelanin synthesis from DHN in early living organisms.

Pursuit of these issues at interdisciplinary level is expected to provide significant advancements into the nature of prebiotic processes with a new focus on PAH-derived species.

\section{Acknowledgements}

This work was financed by FEDER - Fundo Europeu de Desenvolvimento Regional funds through the COMPETE 2020 - Operational Programme for Competitiveness and Internationalisation (POCI), and by Portuguese funds through FCT - Fundação para a Ciência e a Tecnologia in the framework of the project POCI-01-0145-FEDER-029932 (PTDC/FIS-AST/29932/2017). Centro de Química Estrutural acknowledges the financial support of Fundação para a Ciência e Tecnologia (UIDB/00100/2020).

L.R. thanks the European Research Council for funding via the ERC project HYDROMA (grant agreement No. 819587). 


\section{References}

[1] Grimm R. E. and McSween H. Y. (1989) Water and the thermal evolution of carbonaceous chondrite parent bodies. Icarus 82, 244-280.

[2] Bischoff A., Vogel N., and Roszjar J. (2011) The Rumuruti chondrite group. Chemie der Erde - Geochemistry 71, 101-133.

[3] Choe W. H., Huber H., Rubin A. E., Kallemeyn G. W. and Wasson J. T. (2010) Compositions and taxonomy of 15 unusual carbonaceous chondrites. Meteorit. Planet. Sci. 45, 531-554.

[4] Krot A. N., Keil K., Scott E. R. D., Goodrich C. A. and Weisberg M. K. (2014) Classification of meteorites and their genetic relationships. In Meteorites and Cosmochemical Processes, Vol. 1 of Treatise on Geochemistry, Davis A. M. (Ed.), pp.1-63.

[5] McSween H. Y. Jr. and Huss G. R. (2015) In Cosmochemistry. Cambridge University Press.

[6] Alexander C. M. O’D., Cody G. D., De Gregorio B. T., Nittler L. R. and Stroud R. M. (2017) The nature, origin and modification of insoluble organic matter in chondrites, the major source of Earth's C and N. Chemie der Erde-Geochemistry 77, 227-265.

[7] Remusat L. (2015) Organics in primitive meteorites. In Planetary Mineralogy. The European Mineralogical Union, Lee M. R. and Leroux H. (Eds.), pp. 33-65.

[8] Martins Z. (2019) Organic Molecules in Meteorites and Their Astrobiological Significance. In Handbook of Astrobiology, Kolb CRC Press (Ed.), pp. 177-194.

[9] Robert F. and Epstein S. (1982) The concentration and isotopic composition of hydrogen, carbon and nitrogen in carbonaceous meteorites. Geochim. Cosmochim. Acta 46, 81-95.

[10] Alexander C. M. O’D., Fogel M., Yabuta H. and Cody G. D. (2007) The origin and evolution of chondrites recorded in the elemental and isotopic compositions of their macromolecular organic matter. Geochim. Cosmochim. Acta 71, 4380-4403.

[11] Yuen G., Blair N., Des Marais D. J. and Chang S. (1984) Carbon isotope composition of low molecular weight hydrocarbons and monocarboxylic acids from Murchison meteorite. Nature 307, 252-254. 
[12] Krishnamurthy R. V., Epstein S., Cronin J. R., Pizzarello S. and Yuen G. U. (1992) Isotopic and molecular analyses of hydrocarbons and monocarboxylic acids of the Murchison meteorite. Geochim. Cosmochim. Acta 56, 4045-4058.

[13] Cronin J. R., Pizzarello S., Epstein S. and Krishnamurthy R. V. (1993) Molecular and isotopic analyses of the hydroxy acids, dicarboxylic acids, and hydroxydicarboxylic acids of the Murchison meteorite. Geochim. Cosmochim. Acta 57, 4745-4752.

[14] Cooper G. W., Thiemens M. H., Jackson T. L. and Chang S. (1997) Sulfur and Hydrogen Isotope Anomalies in Meteorite Sulfonic Acids. Science 277, 1072-1074.

[15] Tielens A. G. G. M. (1983) Surface chemistry of deuterated molecules. Astron. Astrophys. 119, $177-184$.

[16] Yang J. and Epstein S. (1983) Interstellar organic matter in meteorites. Geochim. Cosmochim. Acta 47, 2199-2216.

[17] Millar T. J., Bennett A. and Herbst E. (1989) Deuterium fractionation in dense interstellar clouds. Astrophys. J. 340, 906-920.

[18] Terzieva R. and Herbst E. (2000) The possibility of nitrogen isotopic fractionation in interstellar clouds. Mon. Not. Royal Astron. Soc. 317, 563-568.

[19] Sandford S. A., Bernstein M. P. and Dworkin J. P. (2001) Assessment of the interstellar processes leading to deuterium enrichment in meteoritic organics. Meteorit. Planet. Sci. 36, 1117-1133.

[20] Robert F. (2003) The D/H ratio in chondrites. Space Sci. Rev. 106, 87-101.

[21] Aléon J. and Robert F. (2004) Interstellar chemistry recorded by nitrogen isotopes in Solar System organic matter. Icarus 167, 424-430.

[22] Remusat L., Palhol F., Robert F., Derenne S. and France-Lanord C. (2006) Enrichment of deuterium in insoluble organic matter from primitive meteorites: A solar system origin? Earth Planet. Sci. Lett. 243, 15-25. 
[23] Remusat L., Robert F., Meibom A., Mostefaoui S., Delpoux O., Binet L., Gourier D. and Derenne S. (2009) Proto-Planetary Disk Chemistry Recorded by D-Rich Organic Radicals in Carbonaceous Chondrites. Astrophys. J. 698, 2087-2092.

[24] Carota E., Botta G., Rotelli L., Di Mauro E. and Saladino R. (2015) Current Advances in Prebiotic Chemistry Under Space Conditions. Curr. Org. Chem. 19, 1963-1979.

[25] Schmitt-Kopplin P., Gabelica Z., Gougeon R.D., Fekete A., Kanawati B., Harir M., Gebefuegi I., Eckel G. and Hertkorn N. (2010) High molecular diversity of extraterrestrial organic matter in Murchison meteorite revealed 40 years after its fall. Proc. Natl. Acad. Sci. U.S.A. 107, 2763-2768.

[26] Aponte J. C., Alexandre M. R., Wang Y., Brearley A. J., Alexander C. M. O’D. and Huang Y. (2011) Effects of secondary alteration on the composition of free and IOM-derived monocarboxylic acids in carbonaceous chondrites. Geochim. Cosmochim. Acta 75, 23092323.

[27] Aponte J. C., Tarozo R., Alexandre M. R., Alexander C. M. O’D. Charnley S. B., Hallmann C., Summons R. E. and Huang Y. (2014) Chirality of meteoritic free and IOM-derived monocarboxylic acids and implications for prebiotic organic synthesis. Geochim. Cosmochim. Acta 131, 1-12.

[28] Aponte J. C., Dworkin J. P. and Elsila J. E. (2014) Assessing the origins of aliphatic amines in the Murchison meteorite from their compound-specific carbon isotopic ratios and enantiomeric composition. Geochim. Cosmochim. Acta 141, 331-345.

[29] Aponte J. C., Dworkin J. P. and Elsila J. E. (2015) Indigenous aliphatic amines in the aqueously altered Orgueil meteorite. Meteorit. Planet. Sci. 50, 1733-1749.

[30] Aponte J. C., McLain H. L., Dworkin J. P. and Elsila J. E. (2016) Aliphatic amines in Antarctic CR2, CM2, and CM1/2 carbonaceous chondrites. Geochim. Cosmochim. Acta 189, 296-311. 
[31] Cooper G., Kimmich N., Belisle W., Sarinana J., Brabham K. and Garrel L. (2001) Carbonaceous meteorites as a source of sugar-related organic compounds for the early Earth. Nature 414, 879-883.

[32] Cronin J. R., Pizzarello S. and Cruikshank D. P. (1988) Organic matter in carbonaceous chondrites, planetary satellites, asteroids and comets. In Meteorites and the early solar system. Kerridhe J. F. and Matthews M. S. (Eds.), University of Arizona Press, pp. 819-857.

[33] Cronin J. R. and Chang S. (1993) Organic matter in meteorites: Molecular and isotopic analyses of the Murchison meteorites. In The chemistry of life's origin. Greenberg J. M., Mendoza-Gomez C. X. and Pirronello V. (Eds.), Kluwer Academic Publishers, The Netherlands, pp. 209-258.

[34] Martins Z. and Sephton M. A. (2009) Extraterrestrial amino acids. In Amino Acids, Peptides and Proteins in Organic Chemistry, Hughes A. B. (Ed.), Wiley, pp. 3-42.

[35] Burton A. S., Stern J. C., Elsila J. E., Glavin D. P. and Dworkin J. P. (2012) Understanding prebiotic chemistry through the analysis of extraterrestrial amino acids and nucleobases in meteorites. Chem. Soc. Rev. 16, 5459-5472.

[36] Glavin D. P., Burton A. S., Elsila J. E., Aponte J. C. and Dworkin J. P. (2020) The Search for Chiral Asymmetry as a Potential Biosignature in our Solar System. Chem. Rev. 120, 46604689.

[37] Shimoyama A., Harada K. and Yanai K. (1985) Amino acids from the Yamato-791198 carbonaceous chondrite from Antarctica. Chem. Lett. 8, 1183-1186.

[38] Shimoyama A. and Harada K. (1984) Amino acid depleted carbonaceous chondrites (C2) from Antarctica. Geochem. J. 18, 281-286.

[39] Shimoyama A. and Ogasawara R. (2002) Dipeptides and diketopiperazines in the Yamato791198 and Murchison carbonaceous chondrites. Orig. Life Evol. Biosph. 32, 165-179.

[40] Shimoyama A., Ponnamperuma C. and Yanai K. (1979) Amino Acids in the Yamato carbonaceous chondrite from Antarctica. Nature 282, 394-396. 
[41] Shimoyama A., Ponnamperuma C. and Yanai K. (1979) Amino acids in the Yamato-74662 meteorite, an Antarctic carbonaceous chondrite. Nat. Inst. Polar Res. 15, 196-205.

[42] Cronin J. R. and Moore C. B. (1971) Amino acid analyses of the Murchison, Murray, and Allende carbonaceous chondrites. Science 172, 1327-1329.

[43] Cronin J. R. and Moore C. B. (1976) Amino acids of the Nogoya and Mokoia carbonaceous chondrites. Geochim. Cosmochim. Acta 40, 853-857.

[44] Cronin J. R., Pizzarello S. and Moore C. B. (1979) Amino acids in an Antarctic carbonaceous chondrite. Science 206, 335-337.

[45] Cronin J. R. and Pizzarello S. (1997) Enantiomeric excesses in meteoritic amino acids. Science 275, 951-955.

[46] Botta O. and Bada J. L. (2002) Extraterrestrial organic compounds in meteorites. Surv. Geophys. 23, 411-467.

[47] Botta O., Glavin D. P., Kminek G. and Bada J. L. (2002) Relative amino acid concentrations as a signature for parent body processes of carbonaceous chondrites. Orig. Life Evol. Biosph. 32, 143-163.

[48] Botta O., Martins Z. and Ehrenfreund P. (2007) Amino acids in Antarctic CM1 meteorites and their relationship to other carbonaceous chondrites. Meteorit. Planet. Sci. 42, 81-92.

[49] Glavin D. P., Dworkin J. P., Aubrey A., Botta O., Doty III J. H., Martins Z. and Bada J. L. (2006) Amino acid analyses of Antarctic CM2 meteorites using liquid chromatography-time of flight-mass spectrometry. Meteorit. Planet. Sci. 41, 889-902.

[50] Glavin D. P., Callahan M. P., Dworkin J. P. and Elsila J. E. (2010) The effects of parent body processes on amino acids in carbonaceous chondrites. Meteorit. Planet. Sci. 45, 1948-1972.

[51] Holzer G. and Oró J. (1979) The organic composition of the Allan Hills carbonaceous chondrite 77306 as determined by pyrolysis-gas chromatography-mass spectrometry and other methods. J. Mol. Evol. 13, 265-270. 
[52] Kotra R. K., Shimoyama A., Ponnamperuma C. and Hare P. E. (1979) Amino acids in a carbonaceous chondrite from Antarctica. J. Mol. Evol. 13, 179-184.

[53] Burton A. S., Glavin D. P., Elsila J. E., Dworkin J. P., Jenniskens P. and Yin Q.-Z. (2014) The amino acid composition of the Sutter's Mill CM2 carbonaceous chondrite. Meteorit. Planet. Sci. 49, 2074-2086.

[54] Burton A. S., Cao T., Nakamura-Messenger K., Berger E. L., Messenger S., Clemett S. J., Aponte J. C. and Elsila J. E. (2016) Organic analysis in the Miller Range 090657 CR2 chondrite: Part 2 amino acid analyses. 47th Lunar and Planetary Science Conference, LPI Contribution No. 1903, p.2987.

[55] Martins Z., Modica P., Zanda Z. and Le Sergeant d'Hendecourt L. (2015) The amino acid and hydrocarbon contents of the Paris meteorite: Insights into the most primitive CM chondrite. Meteorit. Planet. Sci. 50, 926-943.

[56] Martins Z., Alexander C. M. O’D., Orzechowska G. E., Fogel M. L. and Ehrenfreund P. (2007) Indigenous amino acids in primitive CR meteorites. Meteorit. Planet. Sci. 42, 2125 2136.

[57] Martins Z., Hofmann B. A., Gnos E., Greenwood R. C., Verchovsky A., Franchi I. A., Jull A. J. T., Botta O., Glavin D. P., Dworkin J. P. and Ehrenfreund P. (2007) Amino acid composition, petrology, geochemistry, 14C terrestrial age and oxygen isotopes of the Shisr 033 CR chondrite. Meteorit. Planet. Sci. 42, 1581-1595.

[58] Lawless J. G., Kvenvolden K. A. and Peterson E. (1972) Physical Sciences: Evidence for amino acids of extraterrestrial origin in the Orgueil meteorite. Nature 236, 66-67.

[59] Ehrenfreund P., Glavin D. P., Botta O., Cooper G. and Bada J. L. (2001) Extraterrestrial amino acids in Orgueil and Ivuna: Tracing the parent body of CI type carbonaceous chondrites. Proc. Natl. Acad. Sci. U.S.A. 98, 2138-2141. 
[60] Pizzarello S. and Holmes W. (2009) Nitrogen-containing compounds in two CR2 meteorites: 15N composition, molecular distribution and precursor molecules. Geochim. Cosmochim. Acta 73, 2150-2162.

[61] Burton A. S., Elsila J. E., Callahan M. P., Martin M. G., Glavin D. P., Johnson N. M. and Dworkin J. P. (2012) A propensity for $n$ - $\omega$-amino acids in thermally altered Antarctic meteorites. Meteorit. Planet. Sci. 47, 374-386.

[62] Chan H.-S., Martins Z. and Sephton M. A. (2012) Amino acid analyses of type 3 chondrites Colony, Ornans, Chainpur, and Bishunpur. Meteorit. Planet. Sci. 47, 1502-1516.

[63] Burton A. S., McLain H., Glavin D. P., Elsila J. E., Davidson J., Miller K. E., Andronikov A. V., Lauretta D. and Dworkin J. P. (2015) Amino acid analyses of R and CK chondrites. Meteorit. Planet. Sci. 50, 470-482.

[64] Pizzarello S. and Cronin J. R. (2000) Non-racemic amino acids in the Murchison and Murray meteorites. Geochim. Cosmochim. Acta 64, 329-338.

[65] Pizzarello S., Zolensky M. and Turk K. A. (2003) Nonracemic isovaline in the Murchison meteorite: chiral distribution and mineral association. Geochim. Cosmochim. Acta 67, 15891595.

[66] Glavin D. P. and Dworkin J. (2009) Enrichment of the amino acid L-isovaline by aqueous alteration on CI and CM meteorite parent bodies. Proc. Natl. Acad. Sci. U.S.A. 106, 54875492.

[67] Myrgorodska I., Meinert C., Martins Z., le Sergeant d'Hendecourt L. and Meierhenrich U. J. (2016) Quantitative enantioseparation of amino acids by comprehensive two-dimensional gas chromatography applied to non-terrestrial samples. J. Chromatogr. A 1433, 131-136.

[68] Pizzarello S., Schrader D. L., Monroe A. A. and Lauretta D. S. (2012) Large enantiomeric excesses in primitive meteorites and the diverse effects of water in cosmochemical evolution. Proc. Natl. Acad. Sci. USA 109, 11949-11954. 
[69] Kvenvolden K. A., Lawless J., Pering K., Peterson E., Flores J., Ponnamperuma C., Kaplan I. R. and Moore C. (1970) Evidence for extraterrestrial amino-acids and hydrocarbons in the Murchison meteorite. Nature 228, 923-926.

[70] Elsila J. E., Glavin D. P., Dworkin J. P., Martins Z. and Bada J. L. (2012) Inconclusive evidence for nonterrestrial isoleucine enantiomeric excesses in primitive meteorites. Proc. Natl. Acad. Sci. USA 109, E3288.

[71] Cooper G. W., Onwo W. M. and Cronin J. R. (1992) Alkyl phosphonic acids and sulfonic acids in the Murchison meteorite. Geochim. Cosmochim. Acta 56, 4109-4115.

[72] Cataldo F., Ragni P., Iglesias-Groth S. and Manchado A. (2011) Solid state radiolysis of sulphur containing amino acids: cysteine, cystine and methionine. J. Radioanal. Nucl. Chem. 287, 573-580.

[73] Miller S.L. (1953) A production of amino acids under possible primitive earth conditions. Science 117, 528-529.

[74] Martins Z., Price M.C., Goldman N., Sephton M.A. and Burchell M.J. (2013) Shock synthesis of amino acids from impacting cometary and icy planet surface analogues. Nature Geosci. 6, $1045 \mathrm{e} 1049$.

[75] Kitadai N. and Maruyama S. (2018) Origins of building blocks of life: A review. Geosci. Frontiers 9, 1117-1153.

[76] Saladino R., Botta G., Delfino M. and Di Mauro E. (2013) Meteorites as Catalysts for Prebiotic Chemistry. Chem. Eur. J. 19, 16916-16922.

[77] Johnson A.P., Cleaves H.J., Dworkin J.P., Glavin D.P., Lazcano A. and Bada J.L. (2008) The Miller volcanic spark discharge experiment. Science 322, 404.

[78] Jiang L., Dziedzic P., Spacil Z., Zhao G.-L., Nilsson L., Ilag L. L. and Cordova A. (2014) Abiotic synthesis of amino acids and self-crystallization under prebiotic conditions. Sci. Rep. 4, 6769 . 
[79] Marshall W.L. (1994) Hydrothermal synthesis of amino acids. Geochim. Cosmochim. Acta 58, $2099 \mathrm{e} 2106$.

[80] Ménez B., Pisapia C., Andreani M., Jamme F., Vanbellingen Q. P., Brunelle A., Richard L., Dumas P. and Réfrégiers M. (2018) Abiotic synthesis of amino acids in the recesses of the oceanic lithosphere. Nature 564, 59-63.

[81] Saladino R., Di Mauro E. and García-Ruiz J. M. (2019) A Universal Geochemical Scenario for Formamide Condensation and Prebiotic Chemistry. Chem. Eur. J. 25, 3181-3189.

[82] Becker R. S., Hong K. and Hong J. H. (1974) Hot hydrogen atoms reactions of interest in molecular evolution and interstellar chemistry. J. Mol. Evol. 4, 157e172.

[83] Hennet R. J. C., Holm N. G. and Engel M. H. (1992) Abiotic synthesis of amino acids under hydrothermal conditions and the origin of life: a perpetual phenomenon? Naturwissenschaften 79, $361 \mathrm{e} 365$.

[84] Peltzer E. T., Bada J. L., Schlesinger G. and Miller S. L. (1984) The chemical conditions on the parent body of the murchison meteorite: Some conclusions based on amino, hydroxy and dicarboxylic acids. Adv. Space Res. 4, 69-74.

[85] Saladino R., Botta G., Pino S., Costanzo G. and Di Mauro E. (2012) Genetics first or metabolism first? The formamide clue. Chem. Soc. Rev. 41, 5526-5565.

[86] Saitta A. M. and Saija F. (2014) Miller experiments in atomistic computer simulations. Proc. Natl. Ac. Sci. USA 111, 13768-13773.

[87] Saladino R., Carota E., Botta G., Kapralov M., Timoshenko G. N., Rozanov A. Y., Krasavin E. and Di Mauro E. (2015) Meteorite-catalyzed syntheses of nucleosides and of other prebiotic compounds from formamide under proton irradiation. Proc. Natl. Ac. Sci. USA 112, E2746-E2755.

[88] Hudson R. L., Lewis A. S., Moore M. H., Dworkin J. P. and Martin M. G. (2009) Enigmatic isovaline: Investigating the stability, racemization, and formation of a non-biological amino 
acid. Bioastronomy 2007: Molecules, microbes, and extraterrestrial life, ASP Conference Series, 420, 157-162.

[89] Hayatsu R., Studier M. H. and Anders E. (1971) Origin of organic matter in early solar system-IV. Amino acids: Confirmation of catalytic synthesis by mass spectrometry, Geochim. Cosmochim. Acta 35, 939-951.

[90] Friedmann N. and Miller S.L. (1969) Phenylalanine and tyrosine synthesis under primitive Earth conditions. Science 166, $766 \mathrm{e} 767$.

[91] Abd El-Wahab A. H. F., Mohamed H. M., Mohamed El-Agrody A. and Bedair A. H. (2013) The chemical reactivity of naphthols and their derivatives toward $\alpha$-cyanocinnamonitriles and ethyl $\alpha$-cyanocinnamates: A review of synthesis, reactions and applications of naphthopyrano. Eur. J. Chem. 4, 467-483.

[92] Sephton M. A. (2002) Organic Compounds in Carbonaceous Meteorites. Nat. Prod. Rep. 19, $292-311$.

[93] Naraoka H., Shimoyama A., Komiya M., Yamamoto H. and Harada K. (1988) Hydrocarbons in the Yamato-791198 carbonaceous chondrite from Antarctica. Chem. Lett. 17, 831-834.

[94] Shimoyama A., Naraoka H., Komiya M. and Harada K. (1989) Analyses of carboxylic acids and hydrocarbons in Antarctic carbonaceous chondrites, Yamato-74662 and Yamato-793321. Geochem. J. 23, 181-193.

[95] Pering K. L. and Ponnamperuma C. (1971) Aromatic hydrocarbons in the Murchison meteorite. Science 173, 237-239.

[96] Studier M. H., Hayatsu R. and Anders E. (1972) Origin of organic matter in early solar system - V. Further studies of meteoritic hydrocarbons and a discussion of their origin. Geochim. Cosmochim. Acta 36, 189-215.

[97] d'Ischia M., Manini P., Moracci M., Saladino R., Ball V., Thissen H., Evans R. A., Puzzarini C. and Barone V. (2019) Astrochemistry and Astrobiology: Materials Science inWonderland? Int. J. Mol. Sci. 20, 4079-. 
[98] Elsila J. E., de Leon N. P., Buseck P. R. and Zare R. N. (2005) Alkylation of polycyclic aromatic hydrocarbons in carbonaceous chondrites. Geochim. Cosmochim. Acta 69, 13491357.

[99] Altwegg K., Balsiger H., Berthelier J. J., Bieler A., Calmonte U., Fuselier S. A., Goesmann F., Gasc S., Gombosi T. I., Le Roy L., de Keyser J., Morse A., Rubin M., Schuhmann M., Taylor M. G. G. T., Tzou C.-Y. and Wright I. (2017) Organics in comet 67P - a first comparative analysis of mass spectra from ROSINA-DFMS, COSAC and Ptolemy. MNRAS 469, S130-41.

[100] Hadraoui K., Cottin H., Ivanovski S. L., Zapf P., Altwegg K., Benilan Y., Biver N., Della Corte V., Fray N., Lasue J., Merouane S., Rotundi A. and Zakharov V. (2019) Distributed glycine in comet 67P/Churyumov-Gerasimenko. $A \& A$ 630, A32.

[101] Sandford S. A., Aléon J., Alexander C. M. O’D., Araki T., Bajt S., Baratta G. A., Borg J., Bradley J. P., Brownlee D. E., Brucato J. R., Burchell M. J., Busemann H., Butterworth A., Clemett S. J., Cody G., Colangeli L., Cooper G., D'Hendecourt L., Djouadi Z., Dworkin J. P., Ferrini G., Fleckenstein H., Flynn G. J., Franchi I. A., Fries M., Gilles M. K., Glavin D. P., Gounelle M., Grossemy F., Jacobsen C., Keller L. P., Kilcoyne A. L. D., Leitner J., Matrajt G., Meibom A., Mennella V., Mostefaoui S., Nittler L. R., Palumbo M. E., Papanastassiou D. A., Robert F., Rotundi A., Snead C. J., Spencer M. K., Stadermann F. J., Steele A., Stephan T., Tsou P., Tyliszczak T., Westphal A. J., Wirick S., Wopenka B., Yabuta H., Zare R. N. and Zolensky M. E. (2006) Organics captured from comet 81P/Wild 2 by the Stardust spacecraft. Science 314, 1720-1724.

[102] Elsila J. E., Glavin D. P. and Dworkin J. P. (2009) Cometary glycine detected in samples returned by Stardust. Meteorit. Planet. Sci. 44, 1323-1330.

[103] Clemett S. J., Maechling C. R., Zare R. N., Swan P. D. and Walker R. M. (1993) Identification of complex aromatic molecules in individual interplanetary dust particles. Science 262, 721-725. 
[104] Clemett S. J., Chillier X. D. F., Gillette S., Zare R. N., Maurette M., Engrand C. and Kurat G. (1998) Observation of indigenous polycyclic aromatic hydrocarbons in `giant' carbonaceous Antarctic micrometeorites. Orig. Life Evol. Biosph. 28, 425-448.

[105] Brinton K. L. F., Engrand C., Glavin D. P., Bada J. L. and Maurette M. (1998) A search for extraterrestrial amino acids in carbonaceous Antarctic micrometeorites. Orig. Life Evol. Biosph. 28, 413-424.

[106] Glavin D. P., Matrajt G. and Bada J. L. (2004) Re-examination of amino acids in Antarctic micrometeorites. Adv. Space Res. 33, 106-113.

[107] Matrajt G., Pizzarello S., Taylor S. and Brownlee D. (2004) Concentration and variability of the AIB amino acid in polar micrometeorites: Implications for the exogenous delivery of amino acids to the primitive Earth. Meteorit. Planet. Sci. 39, 1849-1858.

[108] Alexander C. M. O’D., Bowden R., Fogel M. L. and Howard K. T. (2015) Carbonate abundances and isotopic compositions in chondrites. Meteorit. Planet. Sci. 50, 810-833.

[109] Le Guillou C., Bernard S., Brearley A. J. and Remusat L. (2014) Evolution of organic matter in Orgueil, Murchison and Renazzo during parent body aqueous alteration: In situ investigations. Geochim. Cosmochim. Acta 131, 368-392.

[110] De Gregorio B. T., Stroud R. M., Nittler L. R., Alexander C. M. O’D., Bassim N. D., Cody G. D., Kilcoyne A. L. D., Sandford S. A., Milam S. N., Nuevo M. and Zega T. J. (2013) Isotopic and chemical variation of organic nanoglobules in primitive meteorites. Meteorit. Planet. Sci. 48, 904-928.

[111] Le Guillou C. and Brearley A. (2014) Relationships between organics, water and early stages of aqueous alteration in the pristine CR3.0 chondrite met 00426. Geochim. Cosmochim. Acta 131, 344-367.

[112] Kissel J. and Krueger F. R. (1987) The organic component in dust from comet Halley as measured by the PUMA mass spectrometer on board Vega 1. Nature 326, 755-760. 
[113] Fray N., Bardyn A., Cottin H., Baklouti D., Briois C., Engrand C., Fischer H., Hornung K., Isnard R., Langevin Y., Lehto H., Le Roy L., Mellado E. M., Merouane S., Modica P., Orthous-Daunay F.-R., Paquette J., Rynö J., Schulz R., Silén J., Siljeström S., Stenzel O., Thirkell L., Varmuza K., Zaprudin B., Kissel J. and Hilchenbach M. (2017) Nitrogen-tocarbon atomic ratio measured by COSIMA in the particles of comet 67P/ChuryumovGerasimenko. MNRAS 469, S506-S516.

[114] Isnard R., Bardyn A., Fray N., Briois C., Cottin H., Paquette J., Stenzel O., Alexander C., Baklouti D., Engrand C., Orthous-Daunay F. R., Siljeström S., Varmuza K. and Hilchenbach M. (2019) H/C elemental ratio of the refractory organic matter in cometary particles of 67P/Churyumov-Gerasimenko. Astron. Astrophys. 630, A27.

[115] Delsemme A. H. (1991) Nature and history of the organic compounds in comets - an astrophysical view. In Comets in the post-Halley era. Newburn R. L. Jr., Neugebauer M. and Rahe J. (Eds.), 377-428.

[116] Bardyn A., Baklouti D., Cottin H., Fray N., Briois C., Paquette J., Stenzel O., Engrand C., Fischer H., Hornung K., Isnard R., Langevin Y., Lehto H., Le Roy L., Ligier N., Merouane S., Modica P., Orthous-Daunay F.-R., Rynö J., Schulz R., Silén J., Thirkell L., Varmuza K., Zaprudin B., Kissel J. and Hilchenbach M. (2017) Carbon-rich dust in comet 67P/Churyumov-Gerasimenko measured by COSIMA/Rosetta. MNRAS 469, S712-S722.

[117] Choukroun M., Altwegg K., Kührt E., Biver N., Bockelée-Morvan D., Drążkowska J., Hérique A., Hilchenbach M., Marschall R., Pätzold M., Taylor M. G. G. T. and Thomas N. (2020) Dust-to-gas and refractory-to-ice mass ratios of comet 67P/Churyumov-Gerasimenko from Rosetta observations. Space Sci. Rev. 216, 44.

[118] Remusat L., Guan Y., Wang Y. and Eiler J. M. (2010) Accretion and preservation of D-rich organic particles in carbonaceous chondrites: Evidence for important transport in the early Solar System nebula. Astrophys. J. 713, 1048-1058. 
[119] Quirico E., Orthous-Daunay F.-R., Beck P., Bonal L., Briani G., Bourot-Denise M., Montagnac G., Dobrica E., Engrand C., Charon E., Rouzaud J.-N. and Gounelle M. (2011) Pre-accretion heterogeneity of organic matter in types 1 and 2 chondrites. Lunar Planet. Sci. 42, \#2372.

[120] Orthous-Daunay F. R., Quirico E., Beck P., Brissaud O., Dartois E., Pino T. and Schmitt B. (2013) Mid-infrared study of the molecular structure variability of insoluble organic matter from primitive chondrites. Icarus 223, 534-543.

[121] Busemann H., Young A. F., Alexander C. M. O’D., Hoppe P., Mukhopadhyay S. and Nittler L. R. (2006) Interstellar chemistry recorded in organic matter from primitive meteorites. Science 312, 727-730.

[122] Floss C., Le Guillou C. and Brearley A. (2014) Coordinated NanoSIMS and FIB-TEM analyses of organic matter and associated matrix materials in CR3 chondrites. Geochim. Cosmochim. Acta 139, 1-25.

[123] Messenger S. (2000) Identification of molecular cloud material in interplanetary dust particules. Nature 404, 968-971.

[124] Aléon J., Robert F., Chaussidon M. and Marty B. (2003) Nitrogen isotopic composition of macromolecular organic matter in interplanetary dust particules. Geochim. Cosmochim. Acta 67, 3773-3783.

[125] Hayatsu R., Matsuoka S., Scott R. G., Studier M. H. and Anders E. (1977) Origin of organic matter in the early solar system-VII. The organic polymer in carbonaceous chondrites. Geochim. Cosmochim. Acta 41, 1325-1339.

[126] Hayatsu R., Winans R. E., Scott R. G., McBeth R. L., Moore L. P. and Studier M. H. (1980) Phenolic esters in the organic polymer of the Murchison meteorite. Science 207, 1202-1204.

[127] Remusat L., Derenne S. and Robert F. (2005) New insight on aliphatic linkages in the macromolecular organic fraction of Orgueil and Murchison meteorites through ruthenium tetroxide oxidation. Geochim. Cosmochim. Acta 69, 4377-4386. 
[128] Huang Y., Alexandre M. R. and Wang Y. (2007) Structure and isotopic ratios of aliphatic side chains in the insoluble organic matter of the Murchison carbonaceous chondrite. Earth Planet. Sci. Lett. 259, 517-525.

[129] Sephton M. A., Pillinger C. T. and Gilmour I. (2000) Aromatic moieties in meteoritic macromolecular materials: analyses by hydrous pyrolysis and $\delta^{13} \mathrm{C}$ of individual compounds. Geochim. Cosmochim. Acta 64, 321-328.

[130] Remusat L., Derenne S., Robert F. and Knicker H. (2005) New pyrolytic and spectroscopic data on Orgueil and Murchison insoluble organic matter: A different origin than soluble? Geochim. Cosmochim. Acta 69, 3919-3932.

[131] Okumura F. and Mimura K. (2011) Gradual and stepwise pyrolyses of insoluble organic matter from the Murchison meteorite revealing chemical structure and isotopic distribution. Geochim. Cosmochim. Acta 75, 7063-7080.

[132] Sephton M. A. (2013) Aromatic units from the macromolecular material in meteorites: Molecular probes of cosmic environments. Geochim. Cosmochim. Acta 107, 231-241.

[133] Derenne S. and Robert F. (2010) Model of molecular structure of the insoluble organic matter isolated from Murchison meteorite. Meteorit. Planet. Sci. 45, 1461-1475.

[134] Garvie L. A. J. and Buseck P. R. (2004) Nanosized carbon-rich grains in carbonaceous chondrite meteorites. Earth Planet. Sci. Lett. 224, 431-439.

[135] Yabuta H., Naraoka H., Sakanishi K. and Kawashima H. (2005) Solid-state 13C NMR characterization of insoluble organic matter from Antarctic CM2 chondrites: Evaluation of the meteoritic alteration level. Meteorit. Planet. Sci. 40, 779-787.

[136] Vinogradoff V., Le Guillou C., Bernard S., Binet L., Cartigny P., Brearley A. J. and Remusat L. (2017) Paris vs. Murchison: Impact of hydrothermal alteration on organic matter in CM chondrites. Geochim. Cosmochim. Acta 212, 234-252. 
[137] Gardinier A., Derenne S., Robert F., Behar F., Largeau C. and Maquet J. (2000) Solid state $\mathrm{CP} / \mathrm{MAS}{ }^{{ }^{3} \mathrm{C}} \mathrm{NMR}$ of the insoluble matter of the Orgueil and Murchison meteorites: quantitative study. Earth Planet. Sci. Lett. 184, 9-21.

[138] Cody G. D., Alexander C. M. O’D. and Tera F. (2002) Solid state ( ${ }^{\prime} \mathrm{H}$ and $\left.{ }^{13} \mathrm{C}\right) \mathrm{NMR}$ spectroscopy of the insoluble organic residue in the Murchison meteorite: A self-consistent quantitative analysis. Geochim. Cosmochim. Acta 66, 1851-1865.

[139] Cody G. D. and Alexander C. M. O’D. (2005) NMR studies of chemical structural variation of insoluble organic matter from different carbonaceous chondrite groups. Geochim. Cosmochim. Acta 69, 1085-1097.

[140] Cody G. D., Heying E., Alexander C. M. O’D., Nittler L. R., Kilcoyne A. L. D., Sandford S. A. and Stroud R. M. (2011) Establishing a molecular relationship between chondritic and cometary organic solids. Proc. Nat. Acad. Sci. 108, 19171-19176.

[141] Levin E. M., Bud'ko S. L., Mao J. D., Huang Y. and Schmidt-Rohr K. (2007) Effect of magnetic particles on NMR spectra of Murchison meteorite organic matter and a polymerbased model system. Solid State Nuclear Magnetic Resonance 31, 63-71.

[142] Cody G. D., Alexander C. M. O’D., Yabuta H., Kilcoyne A. L. D., Araki T., Ade H., Dera P., Fogel M., Militzer B. and Mysen B. O. (2008) Organic thermometry for chondritic parent bodies. Earth Planet. Sci. Lett. 272, 446-455.

[143] Vollmer C., Kepaptsoglou D., Leitner J., Busemann H., Spring N. H., Ramasse Q. M., Hoppe P. and Nittler L. R. (2014) Fluid-induced organic synthesis in the solar nebula recorded in extraterrestrial dust from meteorites. Proc. Nat. Acad. Sci. 111, 15338-15343.

[144] Cody G. D. and Alexander C. M. O’D. (2017) The perplexing nature of nitrogen in chondritic IOM. Lunar Planet. Sci. 48, \#2747.

[145] Orthous-Daunay F.-R., Quirico E., Lemelle L., Beck P., Deandrade V., Simionovici A. and Derenne S. (2010) Speciation of sulfur in the insoluble organic matter from carbonaceous chondrites by XANES spectroscopy. Earth Planet. Sci. Lett. 300, 321-328. 
[146] Herd C. D. K., Blinova A., Simkus D. N., Huang Y., Tarozo R., Alexander C. M. O’D., Gyngard F., Nittler L. R., Cody G. D., Fogel M. L., Kebukawa Y., Kilcoyne A. L. D., Hilts R. W., Slater G. F., Glavin D. P., Dworkin J. P., Callahan M. P., Elsila J. E., De Gregorio B. T. and Stroud R. M. (2011) Origin and evolution of prebiotic organic matter as inferred from the Tagish Lake meteorite. Science 332, 1304-1307.

[147] Alexander C. M. O’D., Cody G. D., Kebukawa Y., Bowden R., Fogel M. L., Kilcoyne A. L. D., Nittler L. R. and Herd C. D. K. (2014) Elemental, isotopic and structural changes in Tagish Lake insoluble organic matter produced by parent body processes. Meteor. Planet. Sci. 49, 503-525.

[148] Wang Y., Huang Y., Alexander C. M. O’D., Fogel M. and Cody G. (2005) Molecular and compound-specific hydrogen isotope analyses of insoluble organic matter from different carbonaceous chondrites groups. Geochim. Cosmochim. Acta 69, 3711-3721.

[149] Remusat L., Palhol F., Robert F., Derenne S. and France-Lanord C. (2006) Enrichment of deuterium in insoluble organic matter from primitive meteorites: A solar system origin? Earth Planet. Sci. Lett. 243, 15-25.

[150] Laurent B., Roskosz M., Remusat L., Robert F., Leroux H., Vezin H., Depecker C., Nuns N. and Lefebvre J.-M. (2015) The deuterium/hydrogen distribution in chondritic organic matter attests to early ionizing irradiation. Nat. Commun. 6, 8567.

[151] Gourier D., Robert F., Delpoux O., Binet L., Vezin H., Moissette A. and Derenne S. (2008) Extreme deuterium enrichment of organic radicals in the Orgueil meteorite: Revisiting the interstellar interpretation? Geochim. Cosmochim. Acta 72, 1914-1923.

[152] Delpoux O., Gourier D., Vezin H., Binet L., Derenne S. and Robert F. (2011) Biradical character of D-rich carriers in the insoluble organic matter of carbonaceous chondrites: A relic of the protoplanetary disk chemistry. Geochim. Cosmochim. Acta 75, 326-336.

[153] Binet L., Gourier D., Derenne S., Robert F. and Ciofini I. (2004) Occurence of abundant diradicaloid moieties in the insoluble organic matter from the Orgueil and Murchison 
meteorites: a fingerprint of its extraterrestrial origin? Geochim. Cosmochim. Acta $\mathbf{6 8}, 881-$ 891.

[154] Laurent B., Roskosz M., Remusat L., Leroux H., Vezin H. and Depecker C. (2014) Isotopic and structural signature of experimentally irradiated organic matter. Geochim. Cosmochim. Acta 142, 522-534.

[155] Kerridge J. F. (1983) Isotopic composition of carbonaceous-chondrite kerogen: evidence for an interstellar origin of organic matter in meteorites. Earth Planet. Sci. Lett. 64, 186-200.

[156] Yang J. and Epstein S. (1984) Relic interstellar grains in Murchison meteorite. Nature 331, 544-547.

[157] Geiss J. and Reeves H. (1981) Deuterium in the solar system. Astron. Astrophys. 93, 189-199.

[158] Draine B. T. (2009) Interstellar dust models and evolutionary implications. In Cosmic Dust Near and Far, Henning T., Grün E. and Steinacker J. (Eds.), pp. 453-473.

[159] Compiègne M., Verstraete L., Jones A., Bernard J.-P., Boulanger F., Flagey N., Le Bourlot J., Paradis D. and Ysard N. (2011) The global dust SED: tracing the nature and evolution of dust with DustEM. Astron. Astrophys. 525, A103.

[160] Pendleton Y. J. and Allamandola L. J. (2002) The organic refractory material in the diffuse interstellar medium: Mid-infrared spectroscopic constraints. Astrophys. J. Suppl. 138, 75-98.

[161] Ehrenfreund P., Robert F. and d'Hendecourt L. (1992) Similarity of the infrared spectrum of an Orgueil organic polymer with interstellar organic compounds in the line of sight towards IRS 7. Adv. Space Res. 12, 53-56.

[162] Alexander C. M. O’D., Cody G. D., Fogel M. and Yabuta H. (2008) Organics in meteorites Solar or interstellar?, In Organic Matter in Space, Kwok S. and Sandford S. A. (Eds.) Cambridge University Press, Hong Kong, pp. 293-297.

[163] Herbst E. and van Dishoeck E. F. (2009) Complex Organic Interstellar Molecules. Ann. Rev. Astron. Astrophys. 47, 427-480. 
[164] Alexander C. M. O’D., Newsome S. D., Fogel M. L., Nittler L. R., Busemann H. and Cody G. D. (2010) Deuterium enrichments in chondritic macromolecular material--Implications for the origin and evolution of organics, water and asteroids. Geochim. Cosmochim. Acta 74, 44174437.

[165] Remusat L., Rouzaud J. N., Charon E., Le Guillou C., Guan Y. and Eiler J. M. (2012) Ddepleted organic matter and graphite in the Abee enstatite chondrite. Geochim. Cosmochim. Acta 96, 319-335.

[166] Hayatsu R. and Anders E. (1981) Organic compounds in meteorites and their origins. In Cosmo- and Geochemistry, Boschke F. L. Ed., 1-37.

[167] Kress M. E. and Tielens A. G. G. M. (2001) The role of Fischer-Tropsch catalysis in solar nebula chemistry. Meteor. Planet. Sci. 36, 75-92.

[168] Llorca J. and Casanova I. (2000) Reaction between $\mathrm{H}_{2}, \mathrm{CO}$, and $\mathrm{H}_{2} \mathrm{~S}$ over Fe, Ni metal in the solar nebula: Experimental evidence for the formation of sulfur-bearing organic molecules and sulfides. Meteor. Planet. Sci. 35, 841-848.

[169] Sekine K., Sugita S., Shido T., Yamamoto T., Iwasawa Y., Kadono T. and Matsui T. (2006) An experimental study on Fischer-Tropsch catalysis: Implications for impact phenomena and nebular chemistry. Meteor. Planet. Sci. 41, 715-730.

[170] Ciesla F. J. and Sandford S. A. (2012) Organic synthesis via irradiation and warming of ice grains in the solar nebula. Science 336, 452-454.

[171] Cleeves L. I., Bergin E. A., Alexander C. M. O’D., Du F., Graninger D., Öberg K. I. and Harries T. J. (2014) The ancient heritage of water ice in the Solar System. Science 345, 15901593.

[172] Cleeves L. I., Bergin E. A., Alexander C. M. O’D., Du F., Graninger D., Öberg K. I. and Harries T. J. (2016) Exploring the origins of deuterium enrichments in solar nebular organics. Astrophys. J. 819, 13. 
[173] Kebukawa Y., Kilcoyne A. L. D. and Cody G. D. (2013) Exploring the potential formation of organic solids in chondrites and comets through polymerization of interstellar formaldehyde. Astrophys. J. 771, 19.

[174] Kebukawa Y. and Cody G. D. (2015) A kinetic study of the formation of organic solids from formaldehyde: Implications for the origin of extraterrestrial organic solids in primitive Solar System objects. Icarus 248, 412-423.

[175] Oba Y., Takano Y., Naraoka H., Furukawa Y., Glavin D. P., Dworkin J. P., Tachibana S. (2020) Extraterrestrial hexamethylenetetramine in meteorites - a precursor of prebiotic chemistry in the inner solar system. Nature Commun. 11, 6243.

[176] Vinogradoff V., Bernard S., Le Guillou C. and Remusat L. (2018) Evolution of interstellar organic compounds under asteroidal hydrothermal conditions. Icarus 305, 358-370.

[177] Tielens A. G. G. M. (2008) Interstellar Polycyclic Aromatic Hydrocarbon Molecules. Ann. Rev. Astron. Astrophys. 46, 289.

[178] Contreras C.S. and Salama F. (2013) Laboratory investigations of polycyclic aromatic hydrocarbon formation and destruction in the circumstellar outflows of carbon stars. Astrophys. J. Suppl. Ser. 208, 6.

[179] Martínez L., Santoro G., Merino P., Accolla M., Lauwaet K., Sobrado J., Sabbah H., Pelaez R. J., Herrero V. J., Tanarro I., Agúndez M., Martín-Jimenez A., Otero R., Ellis G. J., Joblin C., Cernicharo J. and Martín-Gago J. A. (2020) Prevalence of non-aromatic carbonaceous molecules in the inner regions of circumstellar envelopes. Nature Astron. 4, 97-105.

[180] Frenklach M. and Wang H. (1991) Symposium (International) on Combustion, 23, 15591566.

[181] Frenklach M. and Feigelson E. D. (1989) Formation of Polycyclic Aromatic Hydrocarbons in Circumstellar Envelopes. Astrophys. J. 341, 372-384.

[182] Yang T., Kaiser R. I., Troy T. P., Xu B., Kostko O., Ahmed M., Mebel A. M., Zagidullin M. V., Azyazov V. N. (2017) HACAQs Heritage: A Free-Radical Pathway to Phenanthrenein 
Circumstellar Envelopes of Asymptotic Giant Branch Stars. Angew. Chem. Int. Ed. 56, 45154519.

[183] Parker D.S.N., Zhang F., Seol Kim Y., Kaiser R. I., Landera A., Kislov V. V., Mebel A. M. and Tielens A. G. G. M. (2012) Proceed. Natl. Acad. Sci. USA 109, 51.

[184] Zhao L., Kaiser R. I., Xu B., Ablikim U., Ahmed M., Evseev M. M., Bashkirov E. K., Azyazov V. N. and Mebel A. M. (2018) Low-temperature formation of polycyclic aromatic hydrocarbons in Titan's atmosphere. Nature Astron. 2, 973-979.

[185] Bernstein M.P., Dworkin J.P., Sandford S. and Allamandola L. (2001) Ultraviolet irradiation of naphthalene in H2O Ice: implications for meteorites and biogenesis. Met. Planet. Sci. 36, $351-358$

[186] Bernstein M.P., Elsila J.E., Dworkin J.P., Sandford S.A., Allamandola L.J. and Zare R.N. (2002) Side group addition to the PAH coronene by UV photolysis in cosmic ice analogs. Astrophys J. 576, 1115-1120.

[187] Chen Y. J., Nuevo M., Yih T. S., Ip W.-H., Fung H. S., Cheng C. Y., Tsai H. R. and Wu C.Y. R. (2008) Amino acids produced from the ultraviolet/extreme-ultraviolet irradiation of naphthalene in a $\mathrm{H} 2 \mathrm{O}+\mathrm{NH} 3$ ice mixture. MNRAS 384, 605-610.

[188] Bernstein M. P., Sandford S. A., Allamandola L. J., Gillette J. S. B., Clemett S. J. and Zare R. N. (1999) UV irradiation of polycyclic aromatic hydrocarbons in ices: production of alcohols, quinones, and ethers. Science 283, 1135-1138.

[189] Mattioda A. L., Cruz-Diaz G. A., Ging A., Barnhardt M., Boersma C., Allamandola L. J., Schneider T., Vaughn J., Phillips B. and Ricca A. (2020) Formation of Complex Organic Molecules (COMs) from Polycyclic Aromatic Hydrocarbons (PAHs): Implications for ISM IR Emission Plateaus and Solar System Organics. ACS Earth Space Chem. 4, 2227-2245.

[190] Bizzarri B. M., Manini P., Lino V., d’Ischia M., Kapralov M., Krasavin E., Mrzikov K., S`poner J. E., Di Mauro E. and Saladino R. (2020) High-Energy Proton-Beam-Induced 
Polymerization/Oxygenation of Hydroxynaphthalenes on Meteorites and Nitrogen Transfer from Urea: Modeling Insoluble Organic Matter? Chem. Eur. J. 26, 14919 - 14928.

[191] Bernstein M. P., Moore M. H., Elsila J. E., Sandford S. A., Allamandola L. J. and Zare R. N. (2003) Side group addition to the polycyclic aromatic hydrocarbon coronene by proton irradiation in cosmic ice analogs. Astrophys J. 582, L25-L29.

[192] Bouwman J., Cuppen H. M., Bakker A., Allamandola L. J. and Linnartz H. (2010) Photochemistry of the PAH pyrene in water ice: the case for ion-mediated solid-state astrochemistry. Astron. Astrophys. 511, A33.

[193] Bouwman J., Mattioda A. L., Linnartz H. and Allamandola L. J. (2011) Photochemistry of polycyclic aromatic hydrocarbons in cosmic water ice: I. Mid-IR spectroscopy and photoproducts. Astron. Astrophys. 525, A93.

[194] Brussol C., Duane M., Carlier P. and Kotzias D. (1999) Photo-Induced OH reactions of naphthalene and its oxidation products on SiO2. Environ. Sci. Pollut. Res. 6, 138-140.

[195] Zevos N. and Sehested K. (1978) Pulse radiolysis of aqueous naphthalene solutions. J. Phys. Chem. 82, 138-141.

[196] McCollom T. M. and Simoneit B. R. T. (1999) Abiotic formation of hydrocarbons and oxygenated compounds during thermal decomposition of iron oxalate, Orig. Life Evol. Biosph. 29, 167-186.

[197] Williams L. B., Canfield B., Voglesonger K. M. and Holloway J. R. (2005) Organic molecules formed in a primordial womb. Geology 33, 913-916.

[198] Williams L. B., Holloway J. R., Canfield B., Glein C. R., Dick J. M., Hartnett H. E. and Shock E. L. (2011) Birth of Biomolecules from the Warm Wet Sheets of Clays Near Spreading Centers in Earliest Life on Earth: Habitats, Environments and Methods of Detection, Golding S. D. and Glikson M. (Eds.) Springer Science.

[199] Garvie L. A. J. and Buseck P. R. (2006) Carbonaceous materials in the acid residue from the Orgueil carbonaceous chondrite meteorite. Meteorit. Planet. Sci. 41, 633-642. 
[200] Le Guillou C., Rouzaud J.-N., Bonal L., Quirico E., Derenne S. and Remusat L. (2012) High resolution TEM of chondritic carbonaceous matter: Metamorphic evolution and heterogeneity. Meteorit. Planet. Sci. 47, 345-362.

[201] Derenne S., Rouzaud J. N., Clinard C. and Robert F. (2005) Size discontinuity between interstellar and chondritic aromatic structures: A high-resolution transmission electron microscopy study. Geochim. Cosmochim. Acta 69, 3911-3917.

[202] Parker D. S. N. and Kaiser R. I. (2017) On the formation of nitrogen-substituted polycyclic aromatic hydrocarbons (NPAHs) in circumstellar and interstellar environments. Chem. Soc. Rev. 46, 452-463.

[203] Ali A., Sittler Jr. E., Chornay D., Rowe B. and Puzzarini C. (2015) Organic chemistry in Titan's upper atmosphere and its astrobiological consequences: I. views towards Cassini plasma spectrometer (CAPS) and ion neutral mass spectrometer (INMS) experiments in space. Planet. Space Sci. 109-110, 46-63.

[204] https://docs.unity3d.com.

[205] Prota G. (1992) Melanins and Melanogenesis. Academic Press.

[206] Pavan M. E., López N. I. and Pettinari M. J. (2020) Melanin biosynthesis in bacteria, regulation and production perspectives. Appl. Microbiol. Biotechnol. 104, 1357-1370

[207] Gessler N. N., Egorova A. S. and Belozerskaya T. A. (2014) Melanin pigments of fungi under extreme environmental conditions. Appl. Biochem. Microbiol. 50, 105-113.

[208] Schumacher J. (2016) DHN melanin biosynthesis in the plant pathogenic fungus Botrytis cinerea is based on two developmentally regulated key enzyme (PKS)-encoding genes 2016. Mol. Microbiol. 99, 729-48.

[209] Dadachova E., Bryan R. A., Howell R. C., Schweitzer A. D., Aisen P., Nosanchuk J. D. and Casadevall A. (2008) The radioprotective properties of fungal melanin are a function of its chemical composition, stable radical presence and spatial arrangement. Pigment Cell Melanoma Res. 21, 192-199. 
[210] Yabuuchi E. and Ohyama A. (1972) Characterization of "Pyome1anin"-Producing Strains of Pseudomonas aeruginosa. Int. J. Syst. Bacteriol. 22, 53-64.

[211] Denoya C. D., Skinner D. D. and Morgenstern M. R. (1994) A Streptomyces avermitilis gene encoding a 4-hydroxyphenylpyruvic acid dioxygenase-like protein that directs the production of homogentisic acid and an ochronotic pigment in Escherichia coli. J. Bacteriol. 176, 5312-9.

[212] Arias-Barrau E., Olivera E. R., Luengo J. M., Fernández C., Galán B., García J. L., Díaz E. and Miñambres B. (2004) The homogentisate pathway: a central catabolic pathway involved in the degradation of L-phenylalanine, L-tyrosine, and 3-hydroxyphenylacetate in Pseudomonas putida. J. Bacteriol. 186, 5062-77. 2004.

[213] Rodríguez-Rojas A. and Blazquez J. (2009) The Pseudomonas aeruginosa pfpI gene plays an antimutator role and provides general stress protection. J. Bacteriol. 191, 844-850.

[214] Wang Y., Wang H., Cui Z., Chen H., Zhong Z., Kan B. and Zhu J. (2011) The Prevalence of Functional Quorum-Sensing Systems in Recently Emerged Vibrio cholerae Toxigenic Strains. Environ. Microbiol. Rep. 3, 218-222.

[215] Gonyar L. A., Fankhauser S. C. and Goldberg J. B. (2015) Single amino acid substitution in homogentisate 1,2-dioxygenase is responsible for pigmentation in a subset of Burkholderia cepacia complex isolates. Environ. Microbiol. Rep. 7, 180-7.

[216] Wheeler M. H. (1983) Comparisons of fungal melanin biosynthesis in ascomycetous, imperfect and basidiomycetous fungi. Trans. British Mycol. Soc. 81, 29-36.

[217] Bell A. A. and Wheeler M. H. (1986) Biosynthesis and function of fungal melanins. Ann. Rev. Phytopathol. 24, 41-45.

[218] Butler M. J. and Day A. W. (1998) Fungal melanins: a review. Can. J. Microbiol. 44, 11151136.

[219] Henson J. M., Butler M. J. and Day A. W. (1999) The dark side of the mycelium: melanins of phytopathogenic fungi. Ann. Rev. Phytopathol. 37, 447-471. 
[220] Tsai H. F., Wheeler M. H., Chang Y. C. and Kwon-Chung K. J. (1999) A developmentally regulated gene cluster involved in conidial pigment biosynthesis in Aspergillus fumigatus. $J$. Bacteriol. 181, 6469-6477.

[221] Sugareva V., Härtl A., Brock M., Hübner K., Rohde M., Heinekamp T. and Brakhage A. A. (2006) Characterisation of the laccase-encoding gene abr2 of the dihydroxynaphthalene-like melanin gene cluster of Aspergillus fumigatus. Arch. Microbiol. 186, 345-55.

[222] Yoshimoto S., Kosuke I., Atsushi M., Chihiro T. and Kiminori S. (2010) ChMCO1 of Cochliobolus heterostrophus is a new class of metallo-oxidase, playing an important role in DHN-melanization. Mycoscience 51, 327-336.

[223] Lin S. Y., Okuda S., Ikeda K., Okuno T. and Takano Y. (2012) LAC2 Encoding a Secreted Laccase Is Involved in Appressorial Melanization and Conidial Pigmentation in Colletotrichum orbiculare. Mol. Plant Microbe Interact. 25, 1552-1561.

[224] Eisenman H. C. and Casadevall A. (2012) Synthesis and assembly of fungal melanin. Appl. Microbiol. Biotechnol. 93, 931-940.

[225] Schmaler-Ripcke J., Sugareva V., Gebhardt P., Winkler R., Kniemeyer O., Heinekamp T. and Brakhage A. A. (2009) Production of pyomelanin, a second type of melanin, via the tyrosine degradation pathway in Aspergillus fumigatus. Appl. Environ. Microbiol. 75, 493-503.

[226] Prados-Rosales R., Toriola S., Nakouzi A., Chatterjee S., Stark R. Gerfen G., Tumpowsky P., Dadachova E. and Casadevall A. (2015) Structural Characterization of Melanin Pigments from Commercial Preparations of the Edible Mushroom Auricularia auricula. J. Agric. Food Chem. 63, 7326-7332.

[227] Suwannarach N., Kumla J., Watanabe B., Matsui K. and Lumyong S. (2019) Characterization of melanin and optimal conditions for pigment production by an endophytic fungus, Spissiomyces endophytica. PLoS ONE 14, e0222187.

[228] De Angelis F., Arcadi A., Marinelli F., Paci M., Boti D., Pacioni G., and Miranda M. (1996) Partial structures of truffle melanins. Phvtochemistry 43, 1103-1106. 
[229] De Souza R. A., Mukund Kamat N. and Nadkarni V. S. (2018) Purification and characterisation of a sulphur rich melanin from edible mushroom Termitomyces albuminosus Heim. Mycology 9, 296-306.

[230] Harki E., Talou T. and Dargen R. (1997) Purification, characterisation and analysis of melanin extracted from Tuber mehnosporum Vitt. Food Chem. 58, 65-73.

[231] Liu Q., Xiao J., Liu B., Zhuang Y. and Sun L. (2018) Study on the Preparation and Chemical Structure Characterization of Melanin from Boletus griseus. Int. J. Mol. Sci. 19, 3736.

[232] Sajjan S. S., Anjaneya O., Kulkarni G. B., Nayak A. S., Mashetty S. B. and Karegoudar T. B. (2013) Properties and Functions of Melanin Pigment from Klebsiella sp. GSK Korean. $J$. Microbiol. Biotechnol. 41, 60-69.

[233] Gómez-Marín A. M. and Carlos I. S. (2010) Thermal and mass spectroscopic characterization of a sulphur-containing bacterial melanin from Bacillus subtilis. J. Non-Crystal. Sol. 356, 1576-1580.

[234] Vishal G., Pankaj K., Sanju S., Doniya E. M., Sourish B., Satish B. N. and Pramod B. S. (2020) Natural Melanin Produced by the Endophytic Bacillus subtilis 4NP-BL Associated with the Halophyte Salicornia brachiate. J. Agric. Food Chem. 68, 6854-6863.

[235] Meredith P. and Sarna T. (2006) The physical and chemical properties of eumelanin. Pigment Cell Res. 19, 572-94.

[236] Manini P., Lino V., D’Errico G., Reale S., Napolitano A., De Angelis F. and d'Ischia M. (2020) "Blackness" is an index of redox complexity in melanin polymers. Polym. Chem. 11, 5005-5010.

[237] d'Ischia M., Napolitano A., Pezzella A., Meredith P. and Buehler M. (2020) Melanin Biopolymers: Tailoring Chemical Complexity for Materials Design. Angew. Chemie Int. Ed. 59, 11196-11205. 
[238] Pezzella A., Barra M., Musto A., Navarra A., Alfè M., Manini P., Parisi S., Cassinese A., Criscuolo V. and d'Ischia M. (2015) Stem cell-compatible eumelanin biointerface fabricated by chemically controlled solid state polymerization. Mater. Horiz. 2, 212-220.

[239] Lowrey A. H., Famini G. R., Loumbev V., Wilson L. Y. and Tosk J. M. (1997) Modeling drug-melanin interaction with theoretical linear solvation energy relationships. Pigment Cell Res. 10, 251-6.

[240] Han H., Iakovenko L. and Wilson A. C. (2015) Loss of Homogentisate 1,2-Dioxygenase Activity in Bacillus anthracis Results in Accumulation of Protective Pigment. PLoS ONE 10, e0128967.

[241] Nobutaka F., Masanori F., Yasuo O. and Sueharu H. (2005) Biosynthesis of hexahydroxyperylenequinone melanin via oxidative aryl coupling by cytochrome P-450 in Streptomyces griseus. J. Bacteriol. 187, 8149-55.

[242] Orlandi V. T., Bolognese F., Chiodaroli L., Tolker-Nielsen T. and Barbieri P. (2015) Pigments influence the tolerance of Pseudomonas aeruginosa PAO1 to photodynamically induced oxidative stress. Microbiology 161, 2298-2309.

[243] Vijitha V., Chekidhenkuzhiyil J., Abdulaziz A., Sreelakshmi P. K., Saradavey V., Parameswaran P. S. and Shanta N. (2017) Sponge-Associated Bacteria Produce Noncytotoxic Melanin Which Protects Animal Cells from Photo-Toxicity. Appl. Biochem. Biotechnol. 183, 396-411.

[244] Wang Y. and Casadevall A. (1994) Susceptibility of melanized and nonmelanized Cryptococcus neoformans to nitrogen- and oxygen-derived oxidants. Infect. Immun. 62, 30047.

[245] Dadachova E., Bryan R. A., Huang X. C., Moadel T., Schweitzer A. D., Aisen P., Nosanchuk J. D. and Casadevall A. (2007) Ionizing Radiation Changes the Electronic Properties of Melanin and Enhances the Growth of Melanized Fungi. PLoS One 2, e457. 
[246] Robertson K. L., Mostaghim A., Cuomo C. A., Soto C. M., Lebedev N., Bailey R. F. and Wang Z. (2012) Adaptation of the Black Yeast Wangiella dermatitidis to Ionizing Radiation: Molecular and Cellular Mechanisms. PLoS One 7, e48674.

[247] Hulot G. and Gallet Y. (2003) Do superchrons occur without any palaeomagnetic warning? Earth Planet. Sci. Lett. 210, 191-201.

[248] Davis M., Hut P. and Muller R. A. (1985) Terrestrial Catastrophism: Nemesis or Galaxy? Nature 313, 503.

[249] Casadevall A. (2005) Fungal virulence, vertebrate endothermy, and dinosaur extinction: is there a connection? Fungal Genet. Biol. 42, 98-106.

[250] Pizzarello S., Cooper G. and Flynn g. (2006) The Nature and Distribution of the Organic Material in Carbonaceous Chondrites and Interplanetary Dust Particles. Meteor. Early Solar Syst. II 625-651.

[251] Kitadai N. and Maruyama S. (2018) Origins of building blocks of life: A review. Geosci. Frontiers 9, 1117-1153.

[252] Danger G., Ruf A., Maillard J., Hertzog J., Vinogradoff V., Schmitt-Kopplin P., Afonso C., Carrasco N., Schmitz-Afonso I., d'Hendecourt L. L. S. and Remusat L. (2020) Unprecedented Molecular Diversity Revealed in Meteoritic Insoluble Organic Matter: The Paris Meteorite's Case. Planet. Sci. J. 1, 55.

[253] Potenti S., Manini P., Fornaro T., Poggiali G., Crescenzi O., Napolitano A., Brucato J. R., Barone V. and d'Ischia M. (2018) Solid State Photochemistry of Hydroxylated Naphthalenes on Minerals: Probing Polycyclic Aromatic Hydrocarbon Transformation Pathways under Astrochemically-Relevant Conditions. ACS Earth Space Chem. 2, 977-1000.

[254] Gudipati M. S. and Allamandola L. J. (2003) Facile Generation and Storage of Polycyclic Aromatic Hydrocarbon Ions in Astrophysical Ices. Astrophys. J. Lett. 596, L195.

[255] Mrówczyński R., Bunge A. and Liebscher J. (2014) Chem. Eur. J. 20, 8647-8653. 
[256] Tarabella G., Pezzella A., Romeo A., D'Angelo P., Coppedé N., Calicchio M., d'Ischia M. Mosca R. and Iannotta S. (2013) Irreversible evolution of eumelanin redox states detected by an organic electrochemical transistor: en route to bioelectronics and biosensing. J. Mater. Chem. B 1, 3843.

[257] Michaelian K. (2016) Thermodynamic Dissipation Theory of the Origin and Evolution of Life: Salient characteristics of RNA and DNA and other fundamental molecules suggest an origin of life driven by UV-C light, Self-published. Printed by CreateSpace, Mexico City.

[258] Losi A., Viappiani C. and Crippa P. R. (1993) Quenching of the excited states of 8methoxypsoralen by synthetic Eumelanin. J. Fluoresc. 3, 191-194.

[259] Paleos C. M. (2019) Organization and Compartmentalization by Lipid Membranes Promote Reactions Related to the Origin of Cellular Life. Astrobiology 19, 547-552.

[260] Hong S. (2007) J. Phys. Chem. B 111, 7938-47.

[261] https://docs.unity3d.com

[262] https://sourceforge.net/projects/unitymol/

[263] 'VIVETM | Discover Virtual Reality Beyond Imagination'. https://www.vive.com/us/ (accessed Oct. 29, 2020).

[264] 'Oculus Rift| Oculus'. https://www.oculus.com/rift/ (accessed Act. 28, 2020).

[265] Salvadori A., Frate G. D., Pagliai M., Mancini G. and Barone V. (2016) Immersive virtual reality in computational chemistry: Applications to the analysis of QM and MM data. Int. J. Quantum Chem. 116, 1731-1746.

[266] Lazzari F., Salvadori A., Mancini G. and Barone V. (2020) Molecular Perception for Visualization and Computation: The Proxima Library. J. Chem. Inf. Model. 60, 2668-2672.

[267] Martino M., Salvadori A., Lazzari F., Paoloni L., Nandi S., Mancini G., Barone V. and Rampino S. (2020) Chemical promenades: Exploring potential-energy surfaces with immersive virtual reality. J. Comp. Chem. 41, 1310-1323. 


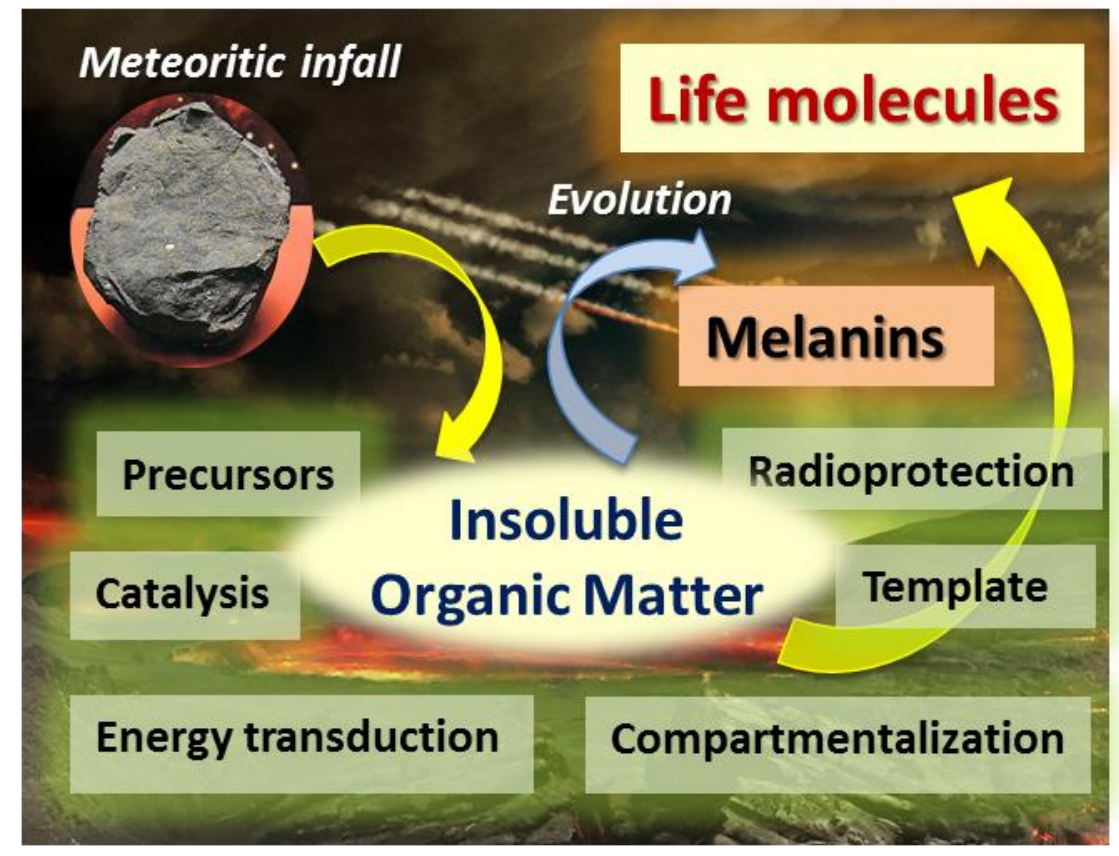




\section{Declaration of interests}

$\bigotimes$ The authors declare that they have no known competing financial interests or personal relationships that could have appeared to influence the work reported in this paper.

$\square$ The authors declare the following financial interests/personal relationships which may be considered as potential competing interests:

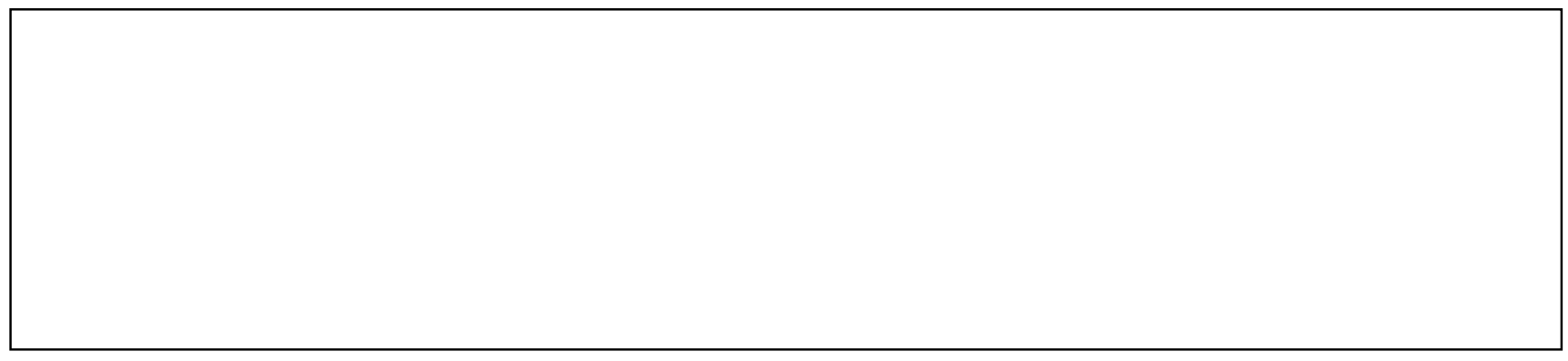

